CONFORMAL GEOMETRY AND DYNAMICS

An Electronic Journal of the American Mathematical Society

Volume 10, Pages 136-158 (July 27, 2006)

S 1088-4173(06)00133-0

\title{
SPIRALS IN THE BOUNDARY OF SLICES OF QUASI-FUCHSIAN SPACE
}

\author{
DAN GOODMAN
}

\begin{abstract}
We prove that the Bers and Maskit slices of the quasi-Fuchsian space of a once-punctured torus have a dense, uncountable set of points in their boundaries about which the boundary spirals infinitely.
\end{abstract}

\section{INTRODUCTION}

Successive zooms into computer pictures of spaces of once-punctured torus groups such as the Maskit slice, for example see Figure 11 suggest that the boundary spirals to an indefinite extent arbitrarily near every point on the boundary. Miyachi Miyachi03 showed that the shape of the boundary of the Maskit, Earle and Bers slices near cusp points is approximately $(2,3)$-cuspidal. Computer plots suggest that if $r / s$ is a very close Farey neighbour of $p / q$, then the direction of the $r / s$-cusp is approximately perpendicular to that of the $p / q$-cusp. By starting at any cusp and repeatedly looking at very close Farey neighbours on the same side, it would seem that for any $n$ we can find a curve within the slice which spirals at least $n$ times around a point very close to the initial cusp in the boundary. This in turn would show that the boundary itself spirals to an indefinite extent arbitrarily near every point on the boundary. Furthermore, it would seem that at the limit point of a sequence of cusps about which the boundary spirals ever more, the boundary would spiral infinitely. The purpose of this paper is to prove that in the case of the Maskit and Bers slices these results hold. In particular, we will prove Theorems 1 and 2 stated below. The definitions related to spiralling are given in Section 2.2.

Theorem 1. The boundaries of the Maskit and Bers slices spiral to an indefinite extent in both directions near every point.

Theorem 2. The boundaries of the Maskit and Bers slices spiral infinitely at an uncountable dense set of points.

This paper is divided into three sections. The first section is the Introduction and contains no rigorous results. It is intended to give an intuitive feeling for what we are aiming to prove, why it is likely to be true and how the proof works. Section 2 contains background theory about quasi-Fuchsian space and slices, more detailed

Received by the editors December 19, 2004 and, in revised form, August 5, 2005.

2000 Mathematics Subject Classification. Primary 37F45; Secondary 37F30.

Key words and phrases. Maskit slice, Bers slice, quasi-Fuchsian space, spiral, once-punctured torus.

The author would like to thank Caroline Series for extensive advice, and the referee for detailed comments on an earlier draft.

(C)2006 American Mathematical Society Reverts to public domain 28 years from publication 

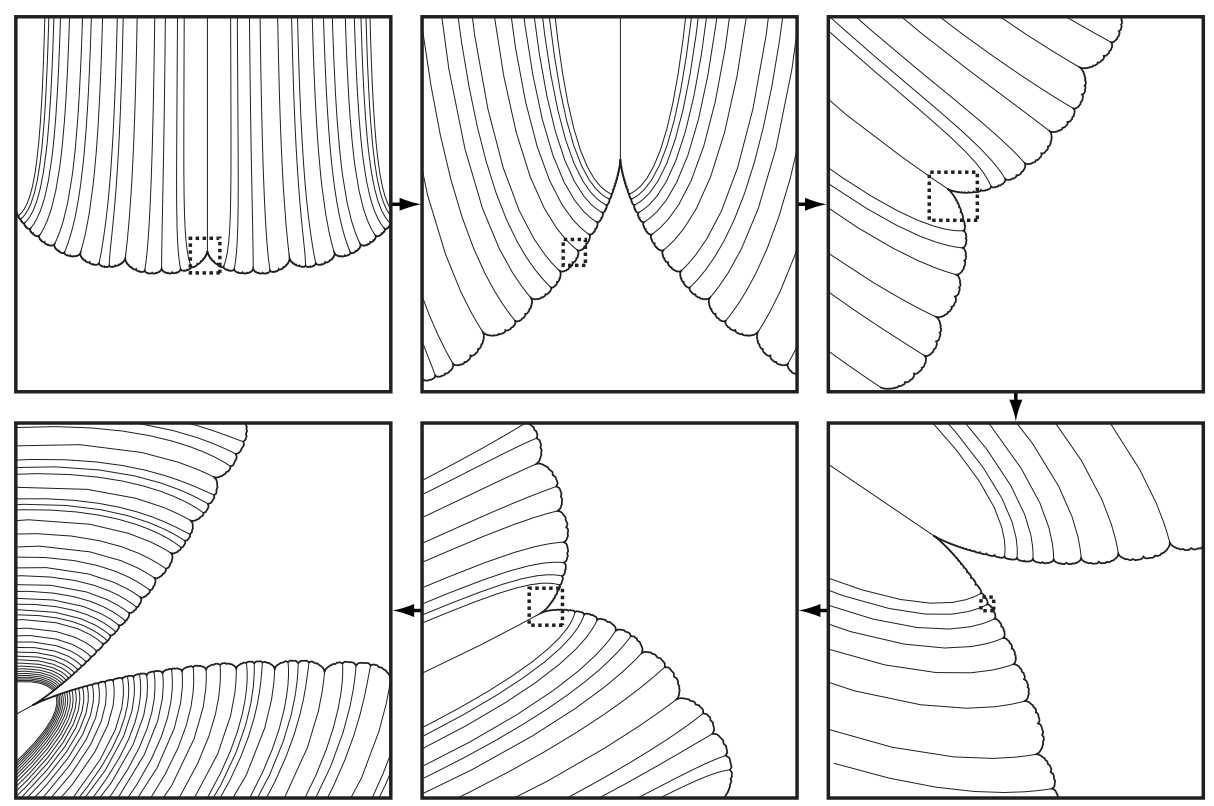

Figure 1. Successive zooms into the Maskit slice suggesting spiralling. The figures go from the top left to the bottom left in a clockwise order. The thick black line in each box is the boundary of the slice; the thinner lines are the pleating rays. The dashed box shows the area being zoomed to in the next box.

background on the structure and shape of cusps (Section 2.1), and the definition of "spiralling" used in this paper (Section 2.2). The final section is the proof. Sections 3.1 and 3.2 are mostly algebra and the details can easily be skipped or just looked over quickly (although the key to the proof is actually an algebraic fact proved in Section 3.2). The difficult part is proving Theorem 1. Theorem 2 is a reasonably simple corollary.

1.1. Motivation. Figure 10 is a picture of the Maskit slice, while Figure 2 shows the Bers slice. These slices are defined in Section 2 In both figures the thick jagged black line is the boundary, and the thinner lines are the pleating rays (Section 2) which end at cusps in the boundary. Figure 1 shows six successive zooms into the Maskit slice. The direction of the main cusp shown gradually rotates counterclockwise from upwards to leftwards. Some of the cusps visible in the last box (the bottom left one) point straight down. If we imagine this procedure to continue indefinitely, and we think about what the boundary must do, it seems it must spiral to an indefinite extent. In other words, the boundary should look a bit like Figure 3 (only the scale of the spirals is so small that it is never visible). In Section 2.2 we will define the exact sense in which it is spiralling.

1.2. Outline. The Maskit and Bers slices are one complex dimensional subset of quasi-Fuchsian once-punctured torus space, which has complex dimension two. By embedding them holomorphically in $\mathbb{C}$, we can think of them simply as subsets of $\mathbb{C}$ bounded by a simple curve. This curve is a homeomorphic image of $\mathbb{R}$ in the 


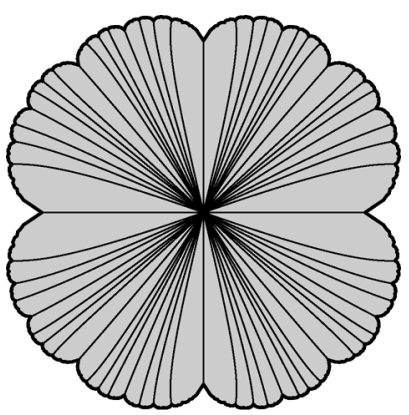

Figure 2. The Bers slice, with the inside shaded gray and pleating rays shown

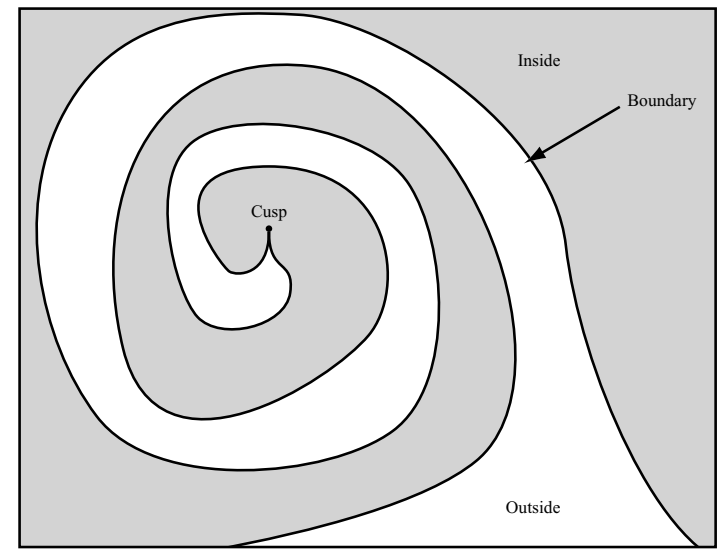

Figure 3. Spiralling - the outside spirals into the cusp, and so does the inside

case of the Maskit slice, or the unit circle $\hat{\mathbb{R}}=\mathbb{R} \cup\{\infty\}$ in the case of the Bers slice. Corresponding to every rational number, there is a cusp in the boundary of the slice. An example is shown in Figure 5. In the case of the Bers slice, $\infty$ also has a corresponding cusp.

Define $\Gamma=\langle X, Y\rangle$ to be the free group on two generators $X$ and $Y$. The geometric meaning will be explained in the next section, but for the moment we only need to understand the combinatorics. The key to understanding the combinatorial structure of the boundary of the Maskit and Bers slices is the following correspondence:

$$
\text { cusps } \longleftrightarrow \text { certain words in } \Gamma \longleftrightarrow \text { rational numbers }
$$

The correspondence between cusps in the boundary of the slice and certain words in $\Gamma$ is geometrically defined in the next section. The correspondence between rational numbers and certain words in $\Gamma$ is defined inductively as follows. Define $W_{-1 / 0}=Y$, $W_{0 / 1}=X$ and $W_{1 / 0}=Y^{-1}$. Now recursively define $W_{p / q}$ by $W_{p / q \oplus r / s}=W_{p / q} W_{r / s}$ where $p s-r q=-1$ (so $p / q<r / s$ ) and $p / q \oplus r / s=(p+r) /(q+s)$. This defines $W_{p / q}$ for all $p / q$, and we call $p / q$ the slope of $W_{p / q}$. The words $W_{p / q}$ correspond to simple closed curves on a once-punctured torus; see Section 2 for details. As an example, we can compute $W_{2 / 3}$ as follows. Firstly, $W_{1 / 1}=W_{0 / 1} W_{1 / 0}$ 


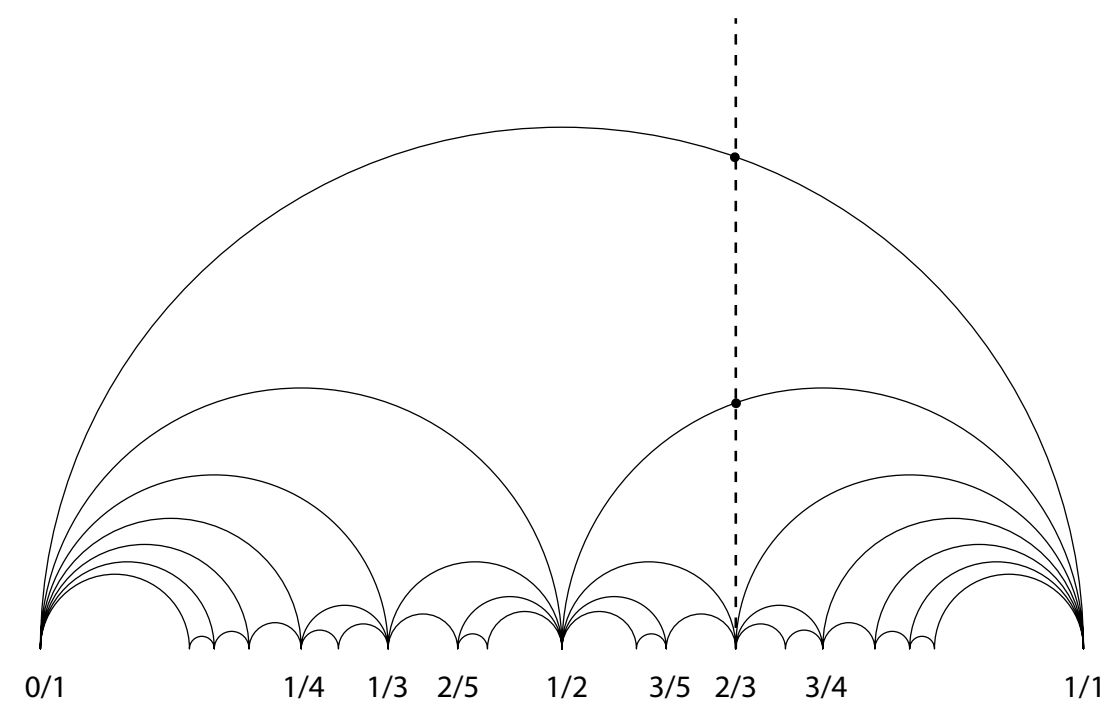

Figure 4 . The Farey graph, showing how to get to $2 / 3$

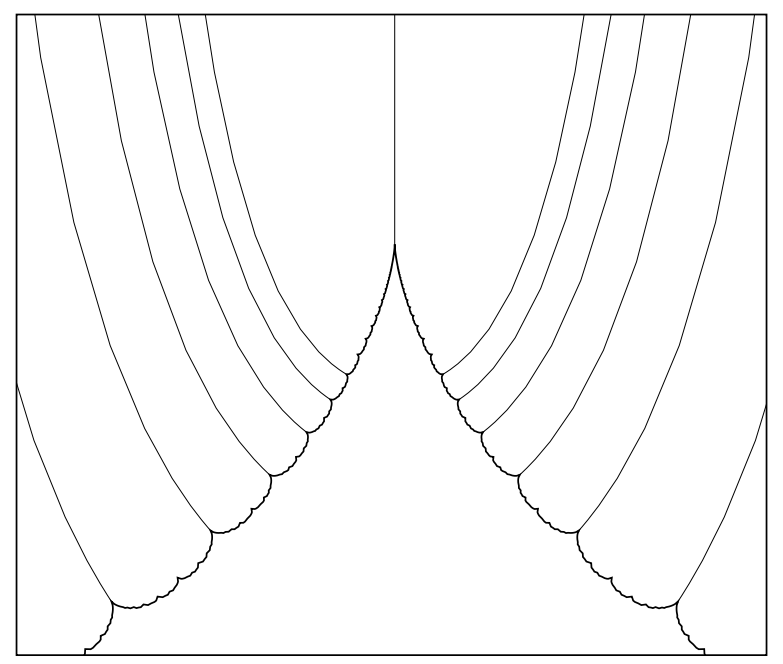

Figure 5. A cusp with some neighbouring cusps in the Maskit slice. The thick line is the boundary; the thinner lines are the pleating rays.

as $0 / 1 \oplus 1 / 0=1 / 1$, so $W_{1 / 1}=X Y^{-1}$. Now $W_{1 / 2}=W_{0 / 1} W_{1 / 1}=X^{2} Y^{-1}$. Finally $W_{2 / 3}=W_{1 / 2} W_{1 / 1}=X^{2} Y^{-1} X Y^{-1}$. This process is illustrated in Figure 4, each edge of the graph intersected by the vertical line ending at $2 / 3$ is a step in the inductive definition of $W_{2 / 3}$. Rational numbers $p / q$ and $r / s$ satisfying $p s-r q= \pm 1$ are said to be Farey neighbours, and two cusps corresponding to $p / q$ and $r / s$ are said to be neighbouring cusps. In the Farey graph, embedded in the hyperbolic upper half plane model in Figure 4, the vertices are rational numbers and two vertices are connected by an edge if they are neighbours. 


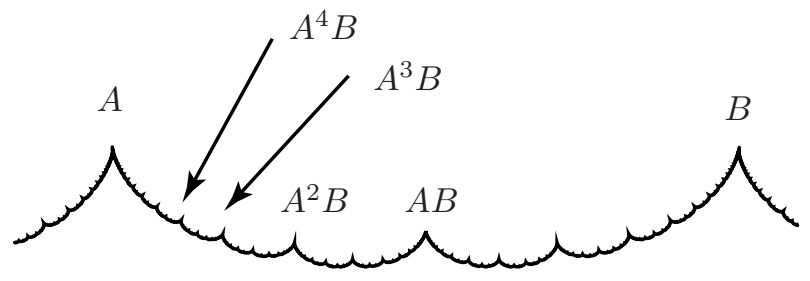

FigURE 6. Enumerating neighbouring cusps

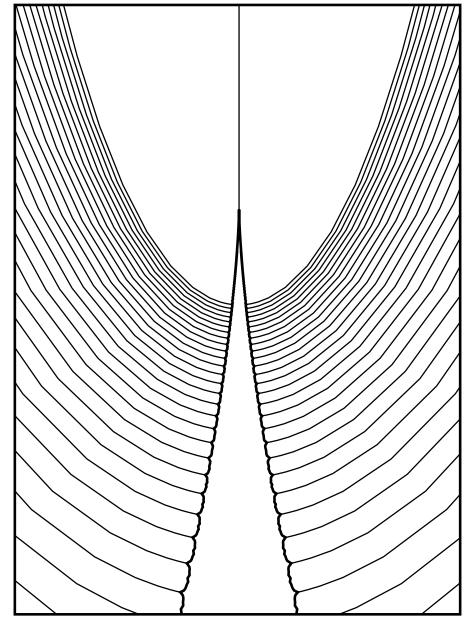

Figure 7. A close-up view of a cusp with some neighbouring cusps in the Maskit slice. The thick line is the boundary; the thinner lines are the pleating rays.

With this combinatorial structure, we can give an outline of the proof of Theorem 1. Suppose that $p / q$ and $r / s$ are two neighbours with $p / q<r / s$. Define $r_{n}=$ $(n p+r) /(n q+s)$, or inductively by $r_{0}=r / s$ and $r_{n+1}=p / q \oplus r_{n}$. It is easy to check that $r_{n}$ and $p / q$ are neighbours. This notation will be used throughout this paper, and we will also write $r_{\infty}=p / q$, so that $r_{n} \rightarrow r_{\infty}$. Suppose that $W_{p / q}=A$ and $W_{r / s}=B$, then the inductive definition above gives us that $W_{r_{n}}=A^{n} B$.

Figure 5 is a close-up of a cusp in the Maskit slice. The thinner less jagged curves are the pleating rays, defined in the next section, which in this diagram end at cusps which are neighbours of the main cusp. It will be shown that the direction of the pleating ray at the cusp is the same as the direction in which the cusp is pointing. This is quite helpful in understanding these figures because it is difficult to see the direction of the smaller cusps themselves. Figure $[$ shows the sequence of neighbouring cusps corresponding to the words $A^{n} B$.

The key lemma in the proof of Theorems 2, 2, and Lemma 7, is that for large $n$, the direction of the $r_{n}$ cusp is approximately perpendicular to the direction of the $p / q$ cusp. This can be seen in Figure 7, an even closer view of a cusp in the Maskit slice. On the assumption of this result, proved largely algebraically in Section 3.2 , the proof of Theorem 1 in Section 3.4 runs roughly as follows. Take any cusp and 


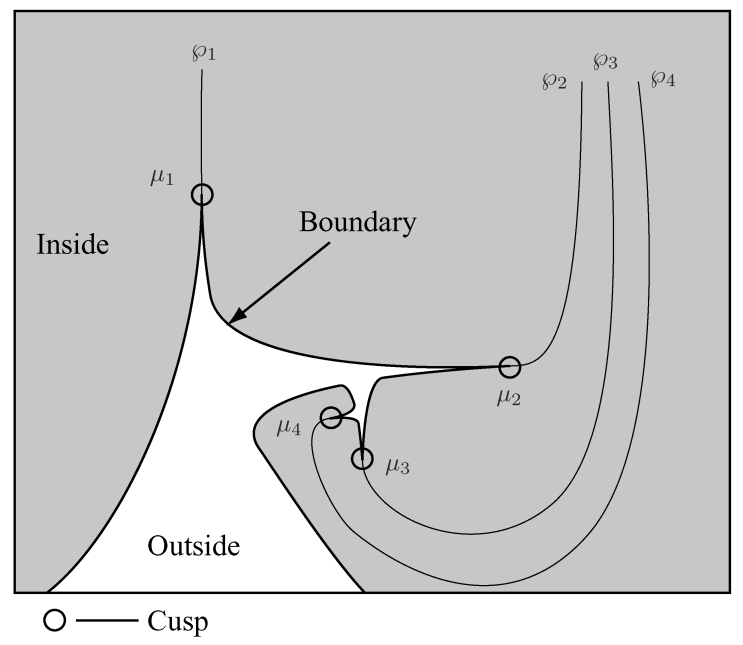

FiguRE 8. The spiral construction argument

look at a very close neighbour. Now look at a close neighbour of this, and so on. Each time you do this, the boundary rotates an extra $\pi / 2$ about the new cusp, so that we can get as much spiralling as we like. Figure 8 illustrates this process with an unrealistic scale (the close neighbouring cusps are in fact very small compared to the main cusp). The curves $\wp_{1}$ to $\wp_{4}$ are the pleating rays ending at the cusps labelled $\mu_{1}$ to $\mu_{4}$.

\section{BACKGROUND AND DEFINITIONS}

Write $\Sigma$ for a fixed once-punctured torus, and $M=\Sigma \times[0,1]$. Define $\mathcal{R}(M)=$ $\operatorname{Hom}\left(\Gamma, \mathrm{PSL}_{2} \mathbb{C}\right) / \mathrm{PSL}_{2} \mathbb{C}$ to be the representation space of a fixed free group on two generators $\Gamma=\langle X, Y\rangle$, modulo conjugation by $\mathrm{PSL}_{2} \mathbb{C}$. Here we think of $\Gamma$ as $\pi_{1} \Sigma$, the fundamental group of $\Sigma$, and $X$ and $Y$ as two generating elements (a meridian and a longitude for example). Similarly, we define $\mathcal{R}_{\mathrm{p}}(M)=\{[\rho] \in$ $\mathcal{R}(M): \rho([X, Y])$ is parabolic $\}$. The set $\mathcal{Q F} \subseteq \mathcal{R}(M)$ consists of those classes of representations whose images are quasi-Fuchsian once-punctured torus groups. In particular, those image groups are discrete and $\rho([X, Y])$ is parabolic for $\rho \in \mathcal{Q F}$. In other words, $\mathcal{Q F} \subseteq \mathcal{R}_{\mathrm{p}}(M)$.

For a representation $\rho \in \operatorname{Hom}\left(\Gamma, \operatorname{PSL}_{2} \mathbb{C}\right)$ such that the conjugacy class $[\rho] \in \mathcal{Q F}$, the complex plane $\mathbb{C}$ is partitioned into three parts, $\Omega_{\rho}^{+}, \Omega_{\rho}^{-}$and $\Lambda_{\rho}$, where $\Omega_{\rho}^{ \pm}$are simply connected regions on which $\rho(\Gamma)$ acts discontinuously and $\Lambda_{\rho}$ is the remainder of $\mathbb{C}$, the limit set. The manifold $M_{\rho}=\left(\mathbb{H}^{3} \cup \Omega_{\rho}^{+} \cup \Omega_{\rho}^{-}\right) / \rho(\Gamma)$ is homeomorphic to $M$. The two ends of this manifold are $\Sigma_{\rho}^{ \pm}=\Omega_{\rho}^{ \pm} / \rho(\Gamma)$, and regarding them as marked Riemann surfaces ( $\rho$ gives the marking), they have Teichmüller parameters $\nu^{ \pm}$. The group $\rho(\Gamma)$ along with an ordered choice of generators is a marked oncepunctured torus group. The group together with the choice of generators is encoded by the representation $\rho$; the ordered choice of generators being $(\rho(X), \rho(Y))$.

We finally complete $\mathcal{Q F}$ to $\overline{\mathcal{Q F}}$ and generalise the Teichmüller parameters $\nu^{ \pm}$to end invariants. First of all, define $\overline{\mathcal{Q F}}$ to be the algebraic closure of $\mathcal{Q F}$ in $\mathcal{R}_{\mathrm{p}}(M)$. 

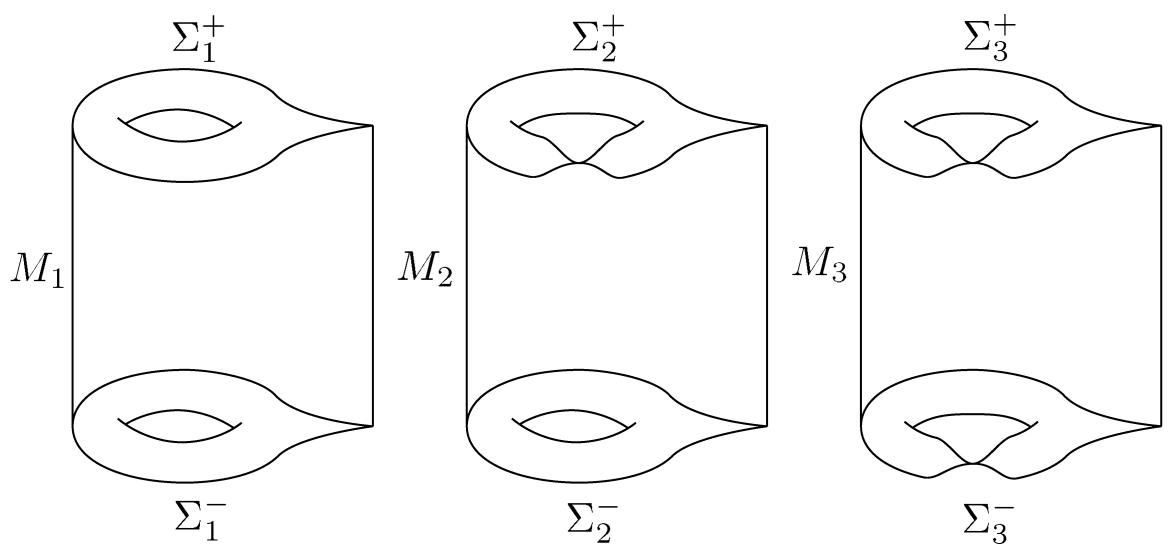

FIgURE 9. Various quasi-Fuchsian manifolds

In a neighbourhood of $\overline{\mathcal{Q F}}, \mathcal{R}_{\mathrm{p}}(M)$ is a smooth complex variety of dimension 2 . The set $\overline{\mathcal{Q F}}$ consists of elements $[\rho] \in \mathcal{R}_{\mathrm{p}}(M)$ such that $\rho(\Gamma)$ is free and discrete.

Writing $\hat{\mathbb{R}}=\mathbb{R} \cup\{\infty\}=S^{1}$, we define $\overline{\mathbb{H}}=\mathbb{H} \cup \hat{\mathbb{R}}$ (or equivalently $\overline{\mathbb{H}}$ is a closed disc), and $\Delta$ to be the diagonal of $\hat{\mathbb{R}} \times \hat{\mathbb{R}}$. For any representation $[\rho] \in \overline{\mathcal{Q F}}$ we can assign a pair of "end invariants" $\left(\nu_{-}, \nu_{+}\right) \in(\overline{\mathbb{H}} \times \overline{\mathbb{H}})-\Delta$. Minsky proved in Minsky99 that there is a continuous bijection $\nu^{-1}:(\overline{\mathbb{H}} \times \overline{\mathbb{H}})-\Delta \rightarrow \overline{\mathcal{Q F}}$ (but that the inverse map $\nu$ is not even continuous). If a marked punctured torus group is in $\partial \mathcal{Q F}=\overline{\mathcal{Q F}}-\mathcal{Q F}$, then either $\nu^{ \pm} \in \mathbb{Q} \cup\{\infty\}$ corresponding to pinching a curve on $\Sigma_{\rho}^{ \pm}$of slope $\nu^{ \pm}$to a point, or $\nu^{ \pm}$is an irrational real corresponding to "pinching a lamination" of slope $\nu^{ \pm}$; see Minsky99 for details.

Figure 9 shows three different quasi-Fuchsian manifolds. The leftmost one is in the interior of $\overline{\mathcal{Q F}}$, both $\Sigma^{+}$and $\Sigma^{-}$are once-punctured tori. The rightmost two are in the boundary $\partial \mathcal{Q F}$. The middle one is pinched at the positive end; that is, the meridian curve on $\Sigma^{+}$has been contracted until it is a single point which is then removed. The surface $\Sigma_{2}^{+}$is then a triply-punctured sphere. If the curve which was pinched corresponds to $W_{p / q} \in \Gamma=\pi_{1} \Sigma$, then the end invariant is $\nu^{+}=p / q$. The right-hand manifold has both ends pinched to triply-punctured spheres. In the diagram, both the + and - ends of the manifold look the same, but in fact this cannot occur. The two curves which are pinched must be different, but this is impossible to illustrate. The right-hand manifold of Figure 9 should be thought of as schematic.

Both the Maskit and Bers slices are defined by holding one end invariant fixed and letting the other vary. Sometimes a Maskit slice is considered to be a slice where every element has end invariants $\left(\nu_{-}, \nu_{+}\right)$and say $\nu_{-}$is fixed. However, we will say that a Maskit slice is any slice where $\nu_{-} \in \mathbb{Q} \cup\{\infty\}$ is fixed and $\nu_{+}$ varies (occasionally called rational Maskit slices), and a Bers slice is any slice where $\nu_{-} \in \mathbb{H}$ is fixed and $\nu_{+}$varies. What we are here calling Maskit slices, McMullen refers to as limit Bers slices in [McMullen98.

In the interior of a Maskit slice, the 3-manifold $M_{\rho}$ has two ends, one of which is a triply-punctured sphere corresponding to the fixed end invariant $\nu_{-}$, and the other is a once-punctured torus corresponding to $\nu_{+}$. This is the middle manifold of Figure 9, On the boundary of a Maskit slice, both ends are triply-punctured 


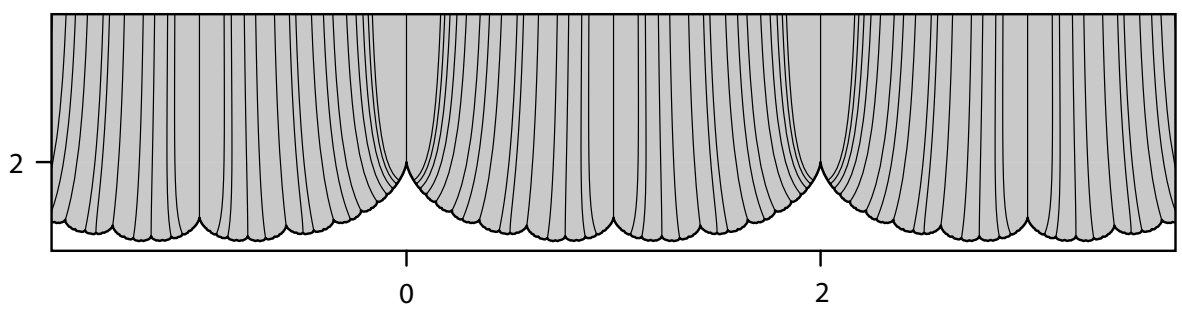

FiguRE 10. The Maskit slice, with pleating rays and interior shaded gray

spheres corresponding to $\nu_{ \pm}$. This is the rightmost manifold of Figure 9. In the interior of a Bers slice, $M_{\rho}$ has two ends, both of which are once-punctured tori corresponding to $\nu_{ \pm}$, the leftmost manifold of Figure 9. On the boundary of a Bers slice, $M_{\rho}$ has one end a once-punctured torus corresponding to $\nu_{-}$and the other end a triply-punctured sphere corresponding to $\nu_{+}$, the middle manifold of Figure 9.

The Maskit slice can be very simply embedded in $\mathbb{C}$ in the following way. Define $g: \mathbb{C} \rightarrow \operatorname{Hom}\left(\Gamma, \mathrm{SL}_{2} \mathbb{C}\right)$ by

$$
g(\mu)(X)=-i\left(\begin{array}{cc}
\mu & 1 \\
1 & 0
\end{array}\right), \quad \text { and } \quad g(\mu)(Y)=\left(\begin{array}{ll}
1 & 2 \\
0 & 1
\end{array}\right) .
$$

Let $\pm: \mathrm{SL}_{2} \mathbb{C} \rightarrow \mathrm{PSL}_{2} \mathbb{C}$ be the quotient map. The map $[g]: \mathbb{C} \rightarrow \mathcal{R}_{\mathrm{p}}(M)$ is defined by setting $[g](\mu)=[ \pm g(\mu)]$, and the set $\overline{\mathcal{M}}=[g]^{-1}(\overline{\mathcal{Q F}}) \cap \mathbb{H}$ is shown in Figure 10. The jagged curve at the bottom is the boundary, and continues periodically in the same fashion with period 2. The slice itself consists of the points on the upper side of this curve. The almost vertical curves coming out of points on the boundary curve are the pleating rays, defined below (see KeenSeries93 and, more generally, KeenSeries04 for more details).

It is possible to embed the Bers slice in $\mathbb{C}$ in a similar way using quadratic differentials.

To cover the case of the Maskit and Bers slices in one, we define a slice of $\overline{\mathcal{Q F}}$ by choosing an injective holomorphic map $f: \mathbb{C} \rightarrow \mathcal{R}_{\mathrm{p}}(M)$. A certain open subset $K \subseteq \mathbb{C}$ will consist of all the interior points of the slice, so that $f$ maps $\bar{K}$ biholomorphically to either the Maskit or Bers slice contained in $\mathcal{R}_{\mathrm{p}}(M)$. In both cases, the boundary $\partial K$ is a simple curve, and $\bar{K}$ is simply connected. Abusing language somewhat, we will also sometimes refer to the set $K$ or $\bar{K}$ as the slice without mentioning $f$.

For a word $W \in \Gamma$ and a slice $f: \mathbb{C} \rightarrow \mathcal{R}_{\mathrm{p}}(M)$, the trace function $\operatorname{Tr}_{W}$ : $\mathbb{C} \rightarrow \mathbb{C}$ is defined by $\operatorname{Tr}_{W}(\mu)=\operatorname{Tr} f(\mu)(W)$. In fact, this only defines $\operatorname{Tr}_{W}$ up to \pm 1 , but if $\mu \in \bar{K}$, then you can choose a sign for $\operatorname{Tr}_{W}$ in a neighbourhood of $f(\mu)$ (or equivalently in a neighbourhood of $\mu$ ) because $\operatorname{Tr} f(\mu)(W) \neq 0$ for a free representation $f(\mu)$. The quantity $\operatorname{Tr}_{W}^{2}$ is always well defined. If $W$ corresponds to a cusp and $\mu_{W}$ is the cusp point on $\partial K$ corresponding to $W$, then $\operatorname{Tr}_{W}^{2}\left(\mu_{W}\right)=4$. The pleating ray corresponding to $W$ is the unique connected subset $\wp_{W} \subseteq K$ of $\left(\operatorname{Tr}_{W}^{2}\right)^{-1}((4, \infty))$ ending at the point $\mu_{W}$. For an explanation of the geometrical significance, see KeenSeries04. These rays can be seen in Figures 10, 12 and 13 , If we choose a sign for $\operatorname{Tr}_{W}$ in a neighbourhood of $\mu_{W}$ and parameterise $\wp_{W}$ by 
$\psi:[2, \infty)$ so that $\operatorname{Tr}_{W} \psi(t)=t$ and differentiate, we get $\psi^{\prime}(t) \operatorname{Tr}_{W}^{\prime}(\psi(t))=1$. In particular, the initial direction of $\wp_{W}$ is $\psi^{\prime}(2)=1 / \operatorname{Tr}_{W}^{\prime}\left(\mu_{W}\right)$. This is also the direction in which the approximately cuspidal boundary points.

2.1. Structure of cusps. In Wright88, David Wright showed, on the basis of conjectures now proven (with one exception, see Section 3.3), that for a cusp $p / q$ in the Maskit slice, the set of cusp points corresponding to Farey neighbouring fractions are approximately $(2,3)$-cuspidal. More precisely, if $p / q$ and $r / s$ are Farey neighbours, and $r_{n}=(n p+r) /(n q+s) \rightarrow r_{\infty}=p / q$, define $\mu_{n}$ to be the cusp point corresponding to $r_{n}$ and $\mu_{\infty}$ to $p / q$. Then he showed that

$$
\mu_{n}=\mu_{\infty}-\frac{\pi^{2}}{A_{1} n^{2}}+\frac{\delta \pi^{2} i}{A_{1} n^{3}}\left(2+B_{0}\right)+O\left(\frac{1}{n^{4}}\right)
$$

where $A_{1}, B_{0}$ and $\delta$ are constants. In particular, $\operatorname{Re}\left(A_{1}\left(\mu_{n}-\mu_{\infty}\right)\right)=-\pi^{2} / n^{2}+$ $O\left(n^{-3}\right), \operatorname{Im}\left(A_{1}\left(\mu_{n}-\mu_{\infty}\right)\right)=C / n^{3}+O\left(n^{-4}\right)$ (for a real constant $C$ ), so that $z_{n}=$ $A_{1}\left(\mu_{n}-\mu_{\infty}\right)$ approximately satisfies an equation of the form $a \operatorname{Re}\left(z_{n}\right)^{3}=\operatorname{Im}\left(z_{n}\right)^{2}$. A curve of points $(x, y)$ is a $(2,3)$-cusp if it satisfies $a x^{3}=y^{2}$.

In Miyachi03, Hideki Miyachi showed that for the Earle, Maskit and Bers slices, there is a neighbourhood of a cusp in which not only the set of neighbouring cusp points, but the whole boundary curve is approximately $(2,3)$-cuspidal. More precisely, he showed that the boundary of any of these slices in the neighbourhood of a cusp is $(2,3)$-cuspidal in the following sense.

Definition 1. A $(2,3)$-cusp curve is a possibly translated and scaled copy of the graph $x^{2}=y^{3}$ in $\mathbb{R}^{2}$. The cusp point is the translated image of $(0,0)$. A curve $\gamma$ is $(2,3)$-cuspidal at a point $P \in \gamma$ if in a neighbourhood of $P$ there are two $(2,3)$-cusp curves with cusp points at $P$ such that $\gamma$ is contained in the region between the two curves. Figure 11] shows this in the case of the boundary of a slice, the two $(2,3)$-cusps are shown dashed, and the boundary curve $\gamma$ is unbroken.

Figure 5 shows an actual plot of a neighbourhood of a cusp in the Maskit slice (with pleating rays).

Below, we give another proof that the boundaries of the Maskit and Bers slices are $(2,3)$-cuspidal at cusps using [ChoiSeries06] to simplify the argument. This also gives us more detailed information about the structure of the cusp. In particular, we find an approximate local parameterisation of the boundary curve which confirms the result of Wright above, and estimate the width of the area of uncertainty between the two bounding (2,3)-cusps. This is summarised in Figure 12. The width estimate will be important later on.

In David Wright's equation (2.1), the constant $A_{1}$ is in fact $\operatorname{Tr}_{\infty}^{\prime}\left(\mu_{\infty}\right)$. It is proved in Miyachi03, and more generally in ChoiSeries06, that:

Proposition 3. $\operatorname{Tr}_{\infty}^{\prime}\left(\mu_{\infty}\right) \neq 0$.

This means that $\operatorname{Tr}_{\infty}$ is a local parameter for the representation space in a neighbourhood of $\mu_{\infty}$. We will prove in Section 3.1, Lemma 5, that

$$
\mu_{n}=\mu_{\infty}-\frac{\pi^{2}}{n^{2} \operatorname{Tr}_{\infty}^{\prime}\left(\mu_{\infty}\right)}+O\left(n^{-3}\right) .
$$

This is consistent with Wright's and Miyachi's results. 


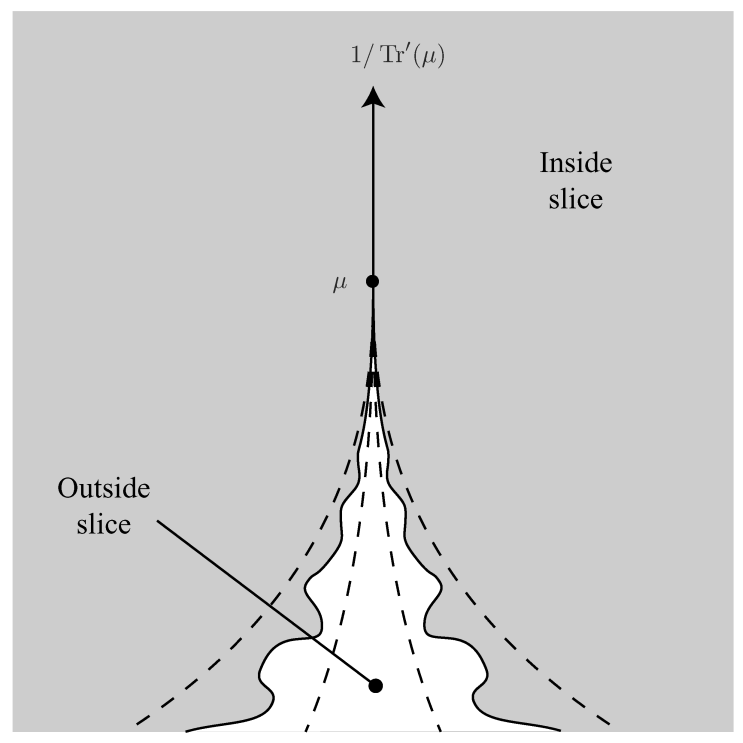

FiguRE 11. Local structure of the boundary near a cusp

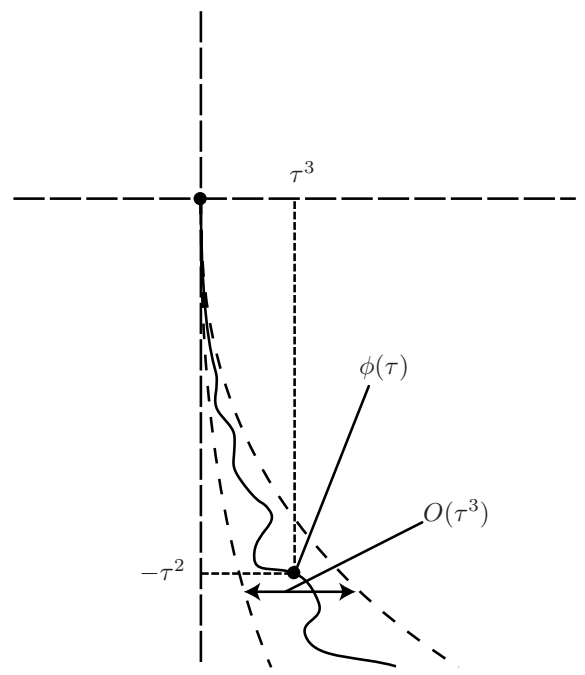

FiguRE 12. Further local structure of the boundary near a cusp

It was mentioned at the end of Section 1.2 that close neighbouring cusps are approximately perpendicular. Figure 13 shows a schematic view of this perpendicularity phenomenon. In this figure the main cusp point is labelled $\mu_{\infty}$ and the sequence of neighbouring cusp points $\mu_{n}$, their directions are $1 / \operatorname{Tr}_{n}^{\prime}\left(\mu_{n}\right)$ (allowing $n=\infty$ ), and their pleating rays are labelled $\wp_{n}$. We shall prove in Lemma 7 and Corollary 8 that

$$
\operatorname{Tr}_{n}^{\prime}\left(\mu_{n}\right)=\frac{\delta n^{3} i \operatorname{Tr}_{\infty}^{\prime}\left(\mu_{\infty}\right)}{\pi^{2}}+O\left(n^{2}\right)
$$




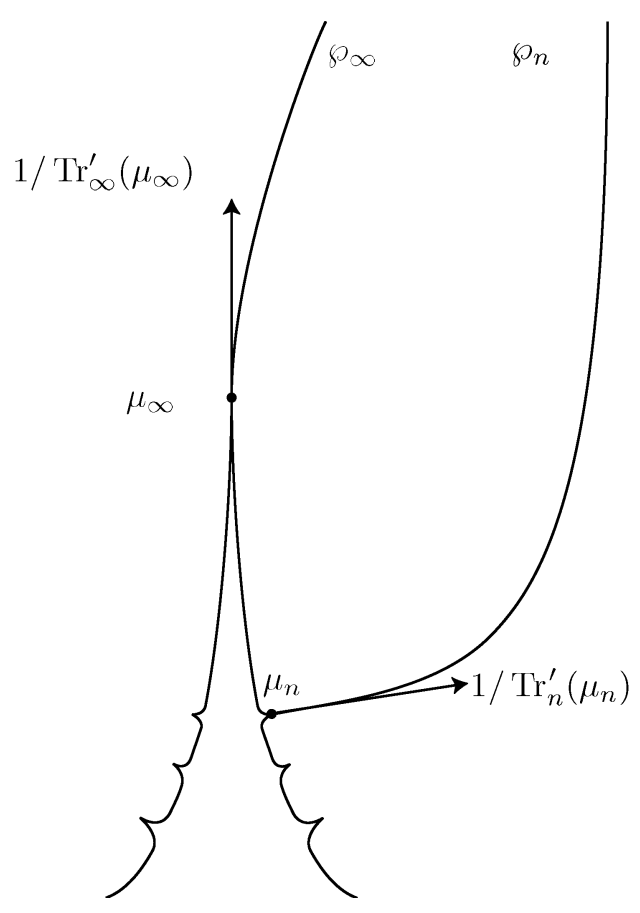

FIGURE 13. Schematic view of the perpendicularity phenomenon

Here, the constant $\delta$ is \pm 1 . Neighbouring cusps on one side of the main cusp will have $\delta=1$, and will have $\delta=-1$ on the other side.

2.2. Spiralling. Defining the term spiralling is just a matter of capturing what is presumably already a shared intuitive idea. In Figure 3, it is clear that both the inside and the outside of the slice "spiral around" the cusp point; and that the boundary, or any curve contained entirely inside or entirely outside the slice, "spirals around" the cusp point as well.

Our definition of spiralling will be relative to a base point $z_{0}$ whose choice will turn out to be irrelevant. Suppose that $K \subseteq \mathbb{C} \cup\{\infty\}$ is any domain with $\partial K$ a simple curve. In particular, $K$ must be simply connected and connected. Fix the base point $z_{0} \in K$. Now choose a point $z_{1} \in \partial K$. Since $\bar{K}-\left\{z_{1}\right\}$ is simply connected and does not contain $z_{1}$, we can define a branch of $\log \left(z-z_{1}\right)$ on this region. In particular, choose the unique branch with $\operatorname{Im} \log \left(z_{0}-z_{1}\right) \in[-\pi, \pi)$. Write $L(z)=\log \left(z-z_{1}\right)$.

Definition 2. The degree of spiralling of any continuous curve $\alpha:[0,1] \rightarrow \bar{K}$ connecting $z_{0}$ to $z_{1}$ with a smooth endpoint and $\alpha^{\prime}(1) \neq 0$ is

$$
\text { sp. } \operatorname{deg} \alpha=\lim _{t \rightarrow 1} \operatorname{Im} L(\alpha(t))-\operatorname{Im} L\left(z_{0}\right) .
$$

The smoothness of $\alpha$ at the endpoint guarantees that sp. $\operatorname{deg} \alpha<\infty$. Such curves may not exist (in particular, if the boundary is spiralling infinitely at that point, see below). 
Definition 3. The degree of spiralling at a boundary point $z_{1}$ is defined to be the set

$$
\text { Sp. } \operatorname{Deg} z_{1}=\left\{\operatorname{sp} . \operatorname{deg} \alpha: \alpha \text { joins } z_{0} \text { to } z_{1}\right\} \text {. }
$$

It turns out that for $z_{1}$ a cusp, all curves ending at $z_{1}$ have approximately the same spiralling degree, and that the exact value of the spiralling degree of a curve $\alpha$ depends only on the direction of the curve at the endpoint, $\alpha^{\prime}(1)$. The following lemma is not necessary, but helps to justify the usefulness of the definition of spiralling given here.

Lemma 4. If $K$ is the Maskit or Bers slice, and $\mu_{p / q} \in \partial K$ is a cusp, then diam Sp. Deg $\mu_{p / q} \leq 2 \pi$. Moreover, two curves $\alpha_{1}$ and $\alpha_{2}$ with smooth endpoints joining the base point $\mu_{0}$ to $\mu_{p / q}$ satisfy $\alpha_{1}^{\prime}(1) /\left|\alpha_{1}^{\prime}(1)\right|=\alpha_{2}^{\prime}(1) /\left|\alpha_{2}^{\prime}(1)\right|$ if and only if either sp. $\operatorname{deg} \alpha_{1}=$ sp. $\operatorname{deg} \alpha_{2}$ or $\left|\mathrm{sp} . \operatorname{deg} \alpha_{1}-\mathrm{sp} . \operatorname{deg} \alpha_{2}\right|=2 \pi$. Assuming sp. $\operatorname{deg} \alpha_{1}<\mathrm{sp} . \operatorname{deg} \alpha_{2}$, the latter situation can only occur if Sp. Deg $\mu_{p / q}=$ [sp. $\operatorname{deg} \alpha_{1}, \operatorname{sp} . \operatorname{deg} \alpha_{2}$ ].

Proof. Let $f: \mathbb{C} \rightarrow \mathcal{R}_{\mathrm{p}}(M)$ be a Maskit or Bers slice with $f(K) \subseteq \overline{\mathcal{Q F}}$ as defined in Section 2. We are considering the boundary point $\mu_{p / q}$, the $p / q$-cusp of a Maskit or Bers slice. The associated $p / q$-word is $W_{p / q} \in \Gamma$. Write $\operatorname{Tr}_{p / q}: U \rightarrow \mathbb{C}$ for the associated trace function, defined by $\operatorname{Tr}_{p / q}(\mu)=\operatorname{Tr}\left(f(\mu)\left(W_{p / q}\right)\right)$, chosen in a neighbourhood $U \subseteq \mathbb{C}$ of $\mu_{p / q}$ so that $\operatorname{Tr}_{p / q}\left(\mu_{p / q}\right)=2$. The base point is $\mu_{0} \in K$ and the function $L$ is the branch of $\log \left(\mu-\mu_{0}\right)$ defined on $\bar{K}$. Proposition 3 asserts that $\operatorname{Tr}_{p / q}^{\prime}\left(\mu_{p / q}\right) \neq 0$. From this we can deduce that there is a smooth curve in the complement of $\bar{K}$ ending at the point $\mu_{p / q}$. Consider the set of points $\mu \in U$ satisfying $\operatorname{Tr}_{p / q}(\mu) \in(0,2)$. Let $\wp_{-}$be the connected component of this set ending at $\mu_{p / q}$. Any such $\mu \in \wp_{-}$cannot be in $\bar{K}$, because for such $\mu$ the word $f(\mu)\left(W_{p / q}\right)$ is elliptic and therefore the associated group $f(\mu)(\Gamma)$ could not be discrete. Moreover, $\wp^{-}$is a smooth curve because it is contained in the real locus of an analytic function.

Choose a sufficiently small ball $B_{\epsilon}$ (of radius $\epsilon$ about $\mu_{p / q}$ ) that $\wp_{-} \cap B_{\epsilon}$ is a simple curve. $\wp_{-} \cap B_{\epsilon}$ connects $\mu_{p / q}$ to the circle $\partial B_{\epsilon}$. Write $C_{\epsilon}=B_{\epsilon}-\wp_{-}$. Given $0<\eta<2 \pi$ we can choose $\epsilon$ sufficiently small that $\wp_{-} \cap B_{\epsilon}$ is contained in a sector of $B_{\epsilon}$ of angle $\eta$. Write $K_{\epsilon}=\left(\bar{K}-\left\{\mu_{p / q}\right\}\right) \cap B_{\epsilon}$. Since $K_{\epsilon} \subseteq C_{\epsilon}$, we have that for any two points $\mu_{1}, \mu_{2} \in K_{\epsilon},\left|\operatorname{Im} L\left(\mu_{1}\right)-\operatorname{Im} L\left(\mu_{2}\right)\right| \leq 2 \pi+\eta$. Letting $\eta \rightarrow 0$ we can easily see that $\mid \operatorname{sp}$. $\operatorname{deg} \alpha_{1}-\operatorname{sp} . \operatorname{deg} \alpha_{2} \mid \leq 2 \pi$. Write $\hat{\alpha}_{i}$ for $\alpha_{i}^{\prime}(1) /\left|\alpha_{i}^{\prime}(1)\right|$. If the spiral degree of $\alpha_{1}$ and $\alpha_{2}$ is the same, then clearly $\hat{\alpha}_{1}=\hat{\alpha}_{2}$. Write $\hat{\wp}_{-}$for the tangent direction of $\wp_{-}$at $\mu_{p / q}$ (which is well defined because $\operatorname{Tr}_{p / q}^{\prime}\left(\mu_{p / q}\right) \neq 0$ ). If sp. $\operatorname{deg} \alpha_{2}=$ sp. $\operatorname{deg} \alpha_{1}+2 \pi$, then $\hat{\alpha}_{1}=\hat{\alpha}_{2}=\hat{\wp}_{-}$. This situation occurs if the two curves $\alpha_{1}$ and $\alpha_{2}$ approach $\mu_{p / q}$ on different sides of $\wp_{-}$. It is also clear that if $0<\mid$ sp. $\operatorname{deg} \alpha_{1}-$ sp. $\operatorname{deg} \alpha_{2} \mid<2 \pi$, then $\hat{\alpha}_{1} \neq \hat{\alpha}_{2}$.

Definition 4. We say that the boundary spirals to an indefinite extent near every point if for any $n \in \mathbb{N}$ and any open neighbourhood $U$ of any point $z_{1}$ in the boundary, there is a point $z_{2} \in U \cap \partial K$ and a curve $\alpha_{2}$ joining $z_{0}$ to $z_{2}$ such that $\left|\operatorname{sp} . \operatorname{deg} \alpha_{2}\right|>n$. We say that the boundary spirals to an indefinite extent in both directions near every point if there are two points $z_{2}, z_{3} \in U \cap \partial K$ and two curves $\alpha_{2}$ and $\alpha_{3}$ joining $z_{0}$ to $z_{2}$ and $z_{3}$ respectively such that sp. $\operatorname{deg} \alpha_{2}>n$ and sp. $\operatorname{deg} \alpha_{3}<-n$.

We extend the definition to infinite spiralling as follows (cf. Pomm92]). 
Definition 5. The boundary spirals infinitely at $z_{1}$ if $\operatorname{Im} L(z)$ is unbounded in every neighbourhood of $z_{1}$.

\section{Proof}

3.1. Cusp structure. The first stage in the proof of Theorem 1 is to prove the facts about the local structure of cusp points described in Section 2.1.

Definition 6. At a given cusp $\mu_{\infty}$ with associated trace function $\operatorname{Tr}_{\infty}$ chosen near $\mu_{\infty}$ so that $\operatorname{Tr}_{\infty}\left(\mu_{\infty}\right)=2$, the sequence $\mu_{n}^{*}$ is defined as follows:

$$
\mu_{n}^{*}=\mu_{\infty}-\frac{\pi^{2}}{n^{2} \operatorname{Tr}_{\infty}^{\prime}\left(\mu_{\infty}\right)} .
$$

Any sequence $\tilde{\mu}_{n}$ satisfying $\tilde{\mu}_{n}=\mu_{n}^{*}+O\left(n^{-3}\right)$ is said to be an approximate cusp sequence.

In particular, we will prove:

Lemma 5. Let $\mu_{n}$ be the sequence of cusps which are neighbours of $\mu_{\infty}$ as in Section 2.1, then the sequence $\mu_{n}$ is an approximate cusp sequence. That is, $\mu_{n}=$ $\mu_{\infty}-\frac{\pi^{2}}{n^{2} \operatorname{Tr}_{\infty}^{\prime}\left(\mu_{\infty}\right)}+O\left(n^{-3}\right)$.

The method of proving this comes from Miyachi03. We use:

Theorem 6 (Pivot theorem, Minsky99). There exist positive constants $\epsilon, c_{1}$ such that, if $\rho: \Gamma \rightarrow \mathrm{PSL}_{2} \mathbb{C}$ is a marked punctured-torus group and $\ell(\alpha) \leq \epsilon$, then

$$
\frac{2 \pi i}{\lambda(\alpha)} \approx \nu_{+}(\alpha)-\overline{\nu_{-}(\alpha)}+i
$$

where " $\approx$ " denotes a bound $c_{1}$ on hyperbolic distance in $\mathbb{H}^{2}$ between the left and right sides.

Here $\alpha$ is an element of the fixed group $\Gamma=\langle X, Y\rangle, \lambda(g)$ is the complex length of the element $g \in \mathrm{PSL}_{2} \mathbb{C}$ defined by the equation $\operatorname{Tr}(g)=2 \cosh \lambda(g) / 2, \lambda(\alpha)=$ $\lambda(\rho(\alpha)), \ell(\alpha)=\operatorname{Re} \lambda(\rho(\alpha))$ and $\nu_{ \pm}(\alpha)$ are the normalised end invariants of the two boundary components of the associated hyperbolic 3-manifold. The normalisation is to choose an element $T \in \mathrm{PSL}_{2} \mathbb{Z}$ sending $p / q$ to $\infty$ where $\alpha$ is the $p / q$-word in $\Gamma$, and to set $\nu_{ \pm}(\alpha)=T\left(\nu_{ \pm}\right)$where $\nu_{ \pm}$are the end invariants or Teichmüller parameters of $\rho$. It is important to note that we think of $\ell(\alpha), \nu_{ \pm}(\alpha)$ and $\lambda(\alpha)$ as functions on $\overline{\mathcal{Q F}}$. In Minsky99, Minsky is considering a fixed representation, whereas we are considering a varying representation. So for example, we define:

$$
\ell(\alpha): \overline{\mathcal{Q F}} \rightarrow \mathbb{R} ; \quad[\rho] \rightarrow \operatorname{Re} \lambda(\rho(\alpha)) .
$$

We are considering a slice embedded in $\mathbb{C}$ via a holomorphic map $f: \mathbb{C} \rightarrow \mathcal{R}_{\mathrm{p}}(M)$, so we can write $[\rho]=f(\mu)$ as a holomorphic function of a complex parameter $\mu$. The cusp $\left[\rho_{\infty}\right]$ (corresponding to pinching $\alpha$ ) corresponds to the point $\mu_{\infty}$. With this understood, we can write

$$
\operatorname{Tr}(\rho(\alpha))=2+\left(\mu-\mu_{\infty}\right) \operatorname{Tr}_{\infty}^{\prime}\left(\mu_{\infty}\right)+O\left(\left(\mu-\mu_{\infty}\right)^{2}\right) .
$$

So we can either think of $\operatorname{Tr}(\rho(\alpha))$ as itself a local coordinate, or we can use this Taylor expansion to find the $\mu$ coordinate. In this section we have chosen the sign of the trace function near $\left[\rho_{\infty}\right]$ so that $\operatorname{Tr}\left(\rho_{\infty}(\alpha)\right)=2$. 
The method is as follows. We wish to estimate the value of $\mu$ giving rise to a representation $\rho$ near $\rho_{\infty}$ and in the boundary of the slice. Using the fact that $\operatorname{Tr}$ is holomorphic and the derivative is nonzero at $\mu_{\infty}$ (by Proposition 3), it suffices to estimate $\operatorname{Tr}(\rho(\alpha))$. Using the formula $\operatorname{Tr}(g)=2 \cosh \lambda(g) / 2$, it suffices to estimate $\lambda(\rho(\alpha))$. We identify the normalised end invariants $\nu_{ \pm}(\alpha)$, and use the pivot theorem to estimate $\lambda(\rho(\alpha))$ (subsequently, just written $\lambda$ ). This gives us an estimate $2 \pi i / \lambda_{0}=\omega_{0}$ of $2 \pi i / \lambda=\omega$, which in turn gives us an estimate $\lambda_{0}$ for $\lambda$.

Proof of Lemma 5. The boundary of the Maskit or Bers slices is a homeomorphic image of $\mathbb{R}$ or $\hat{\mathbb{R}}$, so that in a neighbourhood of the $\alpha$ cusp, $\ell(\alpha) \leq \epsilon$ (as $\ell$ is continuous) and the pivot theorem applies. Writing $B_{r}(z)$ for the hyperbolic ball of radius $r$ about the point $z \in \mathbb{H}$, we have in this case that:

$$
\omega=2 \pi i / \lambda \in B_{c_{1}}\left(\nu_{+}(\alpha)-\overline{\nu_{-}(\alpha)}+i\right) .
$$

Define the point $\omega_{0}=\nu_{+}(\alpha)-\overline{\nu_{-}(\alpha)}+i$ (in the upper half plane).

Let $T \in \mathrm{PSL}_{2} \mathbb{Z}$ be the normalisation so that $T(p / q)=\infty$. Writing $T(p / q)=$ $\frac{a z+b}{c z+d}$, we need to find integers $a, b, c, d$ such that $T(p / q)=\infty$ and $a d-b c=1$. Trying $c=q, d=-p$ we get that $T(p / q)=\infty$ and we need $a, b$ such that $a p+b q=-1$ which can be solved by Euclid's algorithm. This gives:

$$
T(z)=\frac{a}{q}+\frac{1}{q^{2}(z-p / q)} .
$$

So in this notation, $\omega_{0}=T\left(\nu_{+}\right)-\overline{T\left(\nu_{-}\right)}+i$. This in turn gives $\omega_{0}=$ $q^{-2}\left(\nu_{+}-p / q\right)^{-1}+c$ for a constant $c$. An essential point here is that since $\nu_{-}$ is fixed in the Bers and Maskit slices, $T\left(\nu_{-}\right)$is just a constant. Only $\nu_{+}$is varying. We define the symbol $\tau$, which will be used again later, as follows:

$$
\tau=\frac{1}{q^{2}\left(\nu_{+}-p / q\right)} .
$$

With this symbol, we can write $\omega_{0}=\tau+c$. Write $d_{\mathbb{H}}$ for the hyperbolic metric on the upper half plane, and $d_{i \mathbb{H}}$ for the hyperbolic metric on the right-hand half plane, then $\mathbb{H} \rightarrow i \mathbb{H} ; z \mapsto 2 \pi i / z$ is an isometry.

Now define $\lambda_{0}$ by $\omega_{0}=2 \pi i / \lambda_{0}$. The pivot theorem says that $d_{\mathbb{H}}\left(\omega, \omega_{0}\right) \leq c_{1}$, and so using the isometry we get that $d_{i \mathbb{H}}\left(\lambda, \lambda_{0}\right) \leq c_{1}$. The hyperbolic ball of radius $c_{1}$ centred at $\lambda_{0}$ in $i \mathbb{H}$ has Euclidean radius $\operatorname{Re} \lambda_{0} \cdot \sinh c_{1}$, so we get the bound $\left|\lambda-\lambda_{0}\right| \leq 2 \operatorname{Re} \lambda_{0} \cdot \sinh c_{1}$ for the Euclidean distance.

Now $\lambda_{0}=2 \pi i / \omega_{0}=2 \pi i /(\tau+c)=2 \pi i / \tau+O\left(\tau^{-2}\right)$. So Re $\lambda_{0}=O\left(\tau^{-2}\right)$ and hence $d_{\mathbb{E}}\left(\lambda, \lambda_{0}\right)=O\left(\tau^{-2}\right)$. Hence $\lambda=2 \pi i / \tau+O\left(\tau^{-2}\right)$. Using $\operatorname{Tr} W=2 \cosh \lambda(W) / 2$ and the Taylor series for cosh we get that $\operatorname{Tr} \rho \alpha=2-\frac{\pi^{2}}{\tau^{2}}+O\left(\tau^{-3}\right)$. Equating this with equation (3.2), we get that $\mu-\mu_{\infty}=-\frac{\pi^{2}}{\tau^{2} \operatorname{Tr}_{\infty}^{\prime}\left(\mu_{\infty}\right)}+O\left(\tau^{-3}\right)+O\left(\left(\mu-\mu_{\infty}\right)^{2}\right)$.

Explicitly, write $\operatorname{Tr} \rho \alpha=2-\frac{\pi^{2}}{\tau^{2}}+A_{\tau}$, where $\left|A_{\tau}\right| \leq A|\tau|^{-3}$ (for some constant $A)$. Using equation (3.2) we write $\operatorname{Tr} \rho \alpha=2+\left(\mu-\mu_{\infty}\right) \operatorname{Tr}_{\infty}^{\prime}\left(\mu_{\infty}\right)+B_{\mu}$ where $\left|B_{\mu}\right| \leq B\left|\mu-\mu_{\infty}\right|^{2}$ (some $\left.B\right)$. Equating we get that $\mu-\mu_{\infty}=-\frac{\pi^{2}}{\tau^{2} \operatorname{Tr}_{\infty}^{\prime}\left(\mu_{\infty}\right)}+$ $A_{\tau} / \operatorname{Tr}_{\infty}^{\prime}\left(\mu_{\infty}\right)-B_{\mu} / \operatorname{Tr}_{\infty}^{\prime}\left(\mu_{\infty}\right)$. Write $C_{\mu}=\mu-\mu_{\infty}+B_{\mu} / \operatorname{Tr}_{\infty}^{\prime}\left(\mu_{\infty}\right)$ and $C_{\tau}=$ $-\frac{\pi^{2}}{\tau^{2} \operatorname{Tr}_{\infty}^{\prime}\left(\mu_{\infty}\right)}+A_{\tau} / \operatorname{Tr}_{\infty}^{\prime}\left(\mu_{\infty}\right)$. Clearly $C_{\mu}=C_{\tau}$, and $C_{\tau}=O\left(\tau^{-2}\right)$ so we can write $\left|C_{\tau}\right| \leq C|\tau|^{-2}$ (for some $C$ ). It is also clear that for small $\left|\mu-\mu_{\infty}\right|$ we have 
$\left|C_{\mu}\right| \geq D\left|\mu-\mu_{\infty}\right|$ (for some $D$ ). Putting these together we get that $D\left|\mu-\mu_{\infty}\right| \leq$ $C|\tau|^{-2}$. Squaring this, we get that $\left|B_{\mu}\right| \leq B C^{2}|\tau|^{-4} / D^{2}$. So $A_{\tau}-B_{\mu}=O\left(\tau^{-3}\right)$. In conclusion,

$$
\mu=\mu_{\infty}-\frac{\pi^{2}}{\tau^{2} \operatorname{Tr}_{\infty}^{\prime}\left(\mu_{\infty}\right)}+O\left(\tau^{-3}\right) .
$$

Finally, suppose $p / q$ and $r / s$ are Farey neighbours, and define $r_{n}=\frac{n p+r}{n q+s}, r_{\infty}=$ $p / q$. Then $r_{n} \rightarrow r_{\infty}$ and $r_{n}$ is a Farey neighbour of $r_{\infty}$. In fact, the $r_{n}$ are the fractions corresponding to the sequence of neighbouring cusps visible in Figures 5 and 7 If $\nu_{+}=r_{n}$, then $\tau= \pm(n+s / q)$ depending on whether $p s-r q=\mp 1$. This gives us the estimate,

$$
\mu_{n}=\mu_{\infty}-\frac{\pi^{2}}{n^{2} \operatorname{Tr}_{\infty}^{\prime}\left(\mu_{\infty}\right)}+O\left(n^{-3}\right)
$$

as required.

In fact, we can actually say, by looking at Miyachi's argument in Miyachi03 (in particular, the proof of Proposition 4.3), that we must have $a \tau^{-2}<\operatorname{Re} \lambda<b \tau^{-2}$ for $0<a<b$ and $\operatorname{Im} \lambda=2 \pi / \tau+O\left(\tau^{-2}\right)$. Similarly, we can say that $\operatorname{Re} \operatorname{Tr} \rho \alpha=$ $2-\pi^{2} / \tau^{2}+O\left(\tau^{-3}\right)$, and that there are constants $0<a<b$ such that $a \tau^{-3}<$ $|\operatorname{Im} \operatorname{Tr} \rho \alpha|<b \tau^{-3}$. This gives the cusps the local structure discussed in Section 2.1. This refinement will be important later on in Section 3.3 .

3.2. Trace derivatives. Suppose now that $\Gamma=\langle X, Y\rangle$ is a fixed free group on two generators $X$ and $Y$, and that $\Gamma$ is generated by $A$ and $B$ so that, in the boundary of the slice, the cusps corresponding to $A$ and $B$ are neighbours. Let $f: \mathbb{C} \rightarrow \mathcal{R}_{\mathrm{p}}(M)$ be a slice as in Section 2 and let $A$ and $B$ be the $p / q$ and $r / s$ words with respect to the generators $X$ and $Y$. Assume that $p / q<r / s$. Defining $r_{n}=(n p+r) /(n q+s)$, we get that the $r_{n}$ word is $A^{n} B$. This is the sequence of neighbouring cusps between the $A$ and $B$ cusps (see Figure 6). If we had that $r / s<p / q$, then this sequence of neighbouring cusps would be $B A^{n}$ and the conclusions would be mostly but not exactly the same, the points at which the conclusions are different will be highlighted. As before, $\operatorname{Tr}_{n}=\operatorname{Tr}_{A^{n} B}$ and $\operatorname{Tr}_{\infty}=\operatorname{Tr}_{A}$. Below, we will make a careful choice of the signs of these functions.

Lemma 7. If a sequence of points $\tilde{\mu}_{n}$ is an approximate cusp sequence, then

$$
\operatorname{Tr}_{n}^{\prime}\left(\tilde{\mu}_{n}\right)=\frac{ \pm n^{3} i \operatorname{Tr}_{\infty}^{\prime}\left(\mu_{\infty}\right)}{\pi^{2}}+O\left(n^{2}\right)
$$

In particular, since the sequence of neighbouring cusp points $\mu_{n}$ is an approximate cusp sequence, then by Lemma 5 we have:

Corollary 8. For the sequence of neighbouring cusps,

$$
\operatorname{Tr}_{n}^{\prime}\left(\mu_{n}\right)=\frac{ \pm n^{3} i \operatorname{Tr}_{\infty}^{\prime}\left(\mu_{\infty}\right)}{\pi^{2}}+O\left(n^{2}\right)
$$

In particular, the initial directions of two close Farey neighbouring pleating rays are approximately perpendicular to one another.

Proof of Lemma 17. The Markov identity states that for elements $A, B \in \mathrm{SL}_{2} \mathbb{C}$ we have that

$$
(\operatorname{Tr} A)^{2}+(\operatorname{Tr} B)^{2}+(\operatorname{Tr} A B)^{2}=\operatorname{Tr} A \cdot \operatorname{Tr} B \cdot \operatorname{Tr} A B
$$


Choose $\operatorname{Tr}_{A}=\operatorname{Tr}_{\infty}$ near $\mu_{\infty}$ so that $\operatorname{Tr}_{\infty} \mu_{\infty}=2$. Now choose a sign for $\operatorname{Tr}_{B}$, and this will determine a sign choice for $\operatorname{Tr}_{A B}$ so that Markov's identity holds in a neighbourhood of $\mu_{\infty}$. We use the following formula, easily proved by induction for $A, B \in \mathrm{SL}_{2} \mathbb{C}$,

$$
\operatorname{Tr} A^{n} B=a e^{n \lambda / 2}+b e^{-n \lambda / 2},
$$

where $a$ and $b$ are constants depending on $A$ and $B$, and $\lambda$ is the complex length of $A$ defined by $\operatorname{Tr} A=2 \cosh \lambda / 2$. (Note that if we were dealing with the case $r / s<p / q$, this formula would be the same because $\operatorname{Tr} A^{n} B=\operatorname{Tr} B A^{n}$.) If $n=0$, we get $a+b=\operatorname{Tr}_{B}$ and if $n=1$, we get $a e^{\lambda / 2}+b e^{-\lambda / 2}=\operatorname{Tr}_{A B}$. Solving these gives

$$
a=\frac{\operatorname{Tr}_{A B} e^{\lambda / 2}-\operatorname{Tr}_{B}}{e^{\lambda}-1}, \quad b=\frac{e^{\lambda} \operatorname{Tr}_{B}-e^{\lambda / 2} \operatorname{Tr}_{A B}}{e^{\lambda}-1} .
$$

With the choice of functions $\operatorname{Tr}_{A}, \operatorname{Tr}_{B}$ and $\operatorname{Tr}_{A B}$ already made, define a choice of sign for $\operatorname{Tr}_{n}$ using the formula $\operatorname{Tr}_{n}=a e^{n \lambda / 2}+b e^{-n \lambda / 2}$. It may or may not be the case with this choice that $\operatorname{Tr}_{n}\left(\mu_{n}\right)=2$. The sign ambiguity this introduces will be resolved in Lemma 9. Also note that since $\operatorname{Tr}_{A B}, \operatorname{Tr}_{B}$ and $\lambda$ are holomorphic functions of $\mu$, so are $a$ and $b$.

Let $\tilde{\mu}_{n}$ be an approximate cusp sequence, that is,

$$
\tilde{\mu}_{n}=\mu_{\infty}-\frac{\pi^{2}}{n^{2} \operatorname{Tr}_{\infty}^{\prime}\left(\mu_{\infty}\right)}+O\left(n^{-3}\right) .
$$

We know that

$$
\operatorname{Tr}_{\infty}(\mu)=2+\left(\mu-\mu_{\infty}\right) T+O\left(\mu-\mu_{\infty}\right)^{2}
$$

where $T$ is defined as $\operatorname{Tr}_{\infty}^{\prime}\left(\mu_{\infty}\right) \in \mathbb{C}$. Thinking of $\lambda$ as a function in $\mu$, so that

$$
\lambda(\mu)=2 \cosh ^{-1} \operatorname{Tr}_{\infty}(\mu) / 2,
$$

we have

$$
\lambda(\mu)=2 \sqrt{\left(\mu-\mu_{\infty}\right) T}+O\left(\mu-\mu_{\infty}\right)^{3 / 2} .
$$

The square roots in the expression above are not a problem. We will only differentiate $\lambda$ at $\tilde{\mu}_{n} \neq \mu_{\infty}$ and the sign ambiguity that is introduced by the use of the square root will be resolved in Lemma 9 .

We start by differentiating equation (3.7) and substituting equation (3.6) to get

$$
\lambda^{\prime}(\mu)=\frac{\left(\operatorname{Tr}_{\infty}\right)^{\prime}(\mu)}{\sqrt{T\left(\mu-\mu_{\infty}\right)+O\left(\mu-\mu_{\infty}\right)^{2}}} .
$$

Substituting equation (3.5) for $\tilde{\mu}_{n}$ in equation (3.8) we get

$$
\lambda\left(\tilde{\mu}_{n}\right)= \pm \frac{2 i \pi}{n}+O\left(n^{-3 / 2}\right)
$$

Similarly we get

$$
\lambda^{\prime}\left(\tilde{\mu}_{n}\right)=\mp \frac{i n T_{n}}{\pi}+O(1)
$$

where $T_{n}=\operatorname{Tr}_{\infty}^{\prime}\left(\tilde{\mu}_{n}\right)$. Since $\operatorname{Tr}_{\infty}$ is holomorphic at $\mu_{\infty}$ and $\tilde{\mu}_{n} \rightarrow \mu_{\infty}$ we have that $T_{n} \rightarrow T$. In fact, $T_{n}=T+O\left(\tilde{\mu}_{n}-\mu_{\infty}\right)=T+O\left(n^{-2}\right)$. Finally, we differentiate equation (3.3) to get

$$
\operatorname{Tr}_{n}^{\prime}=a^{\prime} e^{n \lambda / 2}+b^{\prime} e^{-n \lambda / 2}+a n \lambda^{\prime} / 2 \cdot e^{n \lambda / 2}-b n \lambda^{\prime} / 2 \cdot e^{-n \lambda / 2} .
$$




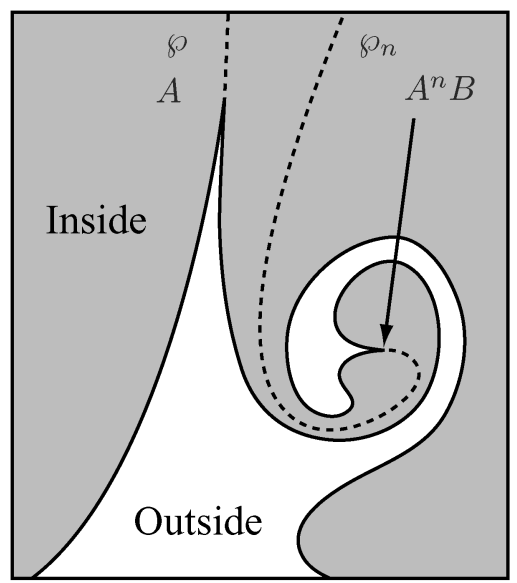

Figure 14. A logically possible monstrosity

Evaluating this at $\tilde{\mu}_{n}$ and using all of the above facts gives, after some simplification, that

$$
\operatorname{Tr}_{n}^{\prime}\left(\tilde{\mu}_{n}\right)= \pm \frac{\left(\operatorname{Tr}_{A B}-\operatorname{Tr}_{B}\right) n^{3} T}{2 \pi^{2}}+O\left(n^{2}\right) .
$$

We now apply Markov's identity. Since $\operatorname{Tr}_{A}\left(\tilde{\mu}_{n}\right)=\operatorname{Tr}_{\infty}\left(\tilde{\mu}_{n}\right)=2+O\left(n^{-2}\right)$ we get $4+\left(\operatorname{Tr}_{B}\right)^{2}+\left(\operatorname{Tr}_{A B}\right)^{2}=2 \operatorname{Tr}_{B} \cdot \operatorname{Tr}_{A B}+O\left(n^{-2}\right)$ which can be factorised to give $\left(\operatorname{Tr}_{B}-\operatorname{Tr}_{A B}\right)^{2}=-4+O\left(n^{-2}\right)$ or $\operatorname{Tr}_{B}-\operatorname{Tr}_{A B}= \pm 2 i+O(1 / n)$. Substituting this in equation (3.13) gives us the required result.

3.3. Curve construction. At this point, we almost have Theorem 1 It would seem as if we could take a cusp, find a close neighbouring cusp approximately perpendicular to it, do the same to this cusp and repeat again and again, each time rotating our view by 90 degrees until we get as much spiralling as we like. There are two problems. First of all, there is a sign ambiguity in $\operatorname{Tr}_{n}^{\prime}\left(\tilde{\mu}_{n}\right)$ in Lemma 7 This might mean that our choice of neighbours might first increase the angle by 90 degrees, and then decrease it by 90 degrees, and so on, so that we end up with no spiralling at all. The other possibility is illustrated in Figure 14, In this case, we would actually rotate the angle by -270 degrees rather than +90 degrees.

In Wright88], David Wright conjectured that the straight line segment between any two neighbouring cusps in the Maskit slice is entirely contained within the slice. This is the conjecture referred to at the beginning of Section 2.1. This conjecture would rule out the possibility of something like Figure 14, Below, we prove something almost as good as Wright's conjecture, at least for the purposes of this paper, that there is an arc between two close neighbouring cusps consisting of an almost straight segment of length $O\left(n^{-3}\right)$ in the direction of the $A^{n} B$ cusp followed by an almost straight segment of length $O\left(n^{-2}\right)$ in the direction of the $A$ cusp. This is also good enough to rule out the possibility of Figure 14.

The construction of this arc is roughly as follows, illustrated in Figure 15 (the $O(-)$ notation refers to the length of the two components of the arc). Near a close neighbouring cusp, the trace derivative satisfies the perpendicularity equation of Lemma 7. Suppose $\operatorname{Tr}_{n}\left(\mu_{n}\right)=2$, and the pleating ray is $\wp_{n}(t)$ parameterised so 


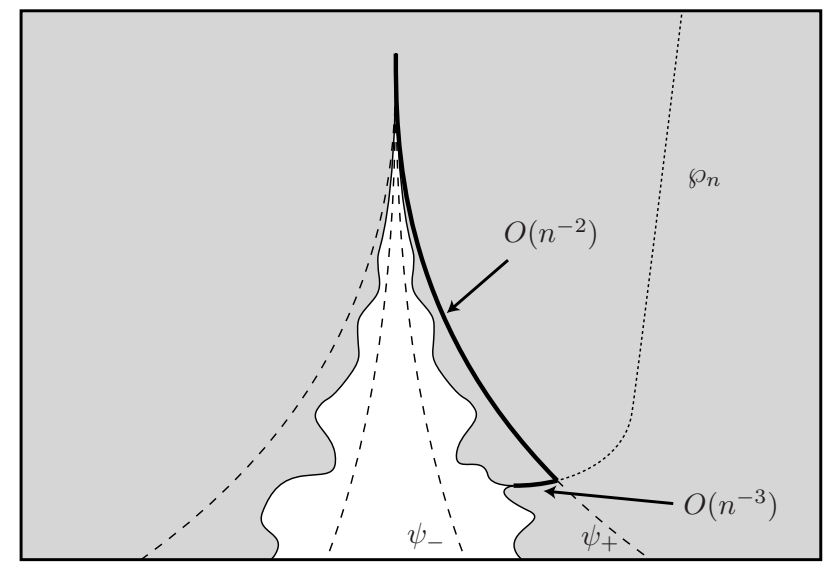

Figure 15. Constructing an almost straight arc between two neighbouring cusps

that $\operatorname{Tr}_{n}\left(\wp_{n}(t)\right)=t$, then the direction of the pleating ray at $\wp_{n}(t)$ is $1 / \operatorname{Tr}_{n}^{\prime}\left(\wp_{n}(t)\right)$ by a simple application of the chain rule. So, at those points $\wp_{n}(t)$ within some small neighbourhood of the $A^{n} B$-cusp, the direction of the pleating ray will be approximately perpendicular to the direction of the pleating ray of the main cusp. It turns out that the size of the neighbourhood of the $A^{n} B$-cusp in which this is true is large enough that the pleating ray reaches one of the bounding $(2,3)$-cusps of the main cusp. The arc is then the initial section of the pleating ray $\wp_{n}$ to the point where it touches one of the bounding $(2,3)$-cusps, followed by the segment of the bounding $(2,3)$-cusp from the intersection point to the main cusp. More explicitly, let $\psi_{+}$be the outer bounding $(2,3)$-cusp curve. The arc we construct consists of the initial segment of $\wp_{n}$ (from $\wp_{n}(2)$ to $\wp_{n}\left(t_{0}\right)$ where $\wp_{n}\left(t_{0}\right)$ is the first point of intersection of $\wp_{n}$ and $\psi_{+}$), followed by the segment of $\psi_{+}$from this intersection point to the main cusp.

Lemma 7 says that for any approximate cusp sequence $\tilde{\mu}_{n}$, we get an equation for $\operatorname{Tr}_{n}^{\prime}\left(\tilde{\mu}_{n}\right)$ depending only on $n$ with an $O\left(n^{2}\right)$ term. Here we are not assuming that $\tilde{\mu}_{n}$ are the neighbouring cusps. Instead, suppose that $\mu_{n}$ is the sequence of cusp neighbours of $\mu_{\infty}$ and $\tilde{\mu}_{n}$ is any sequence with $\tilde{\mu}_{n}=\mu_{n}+O\left(n^{-3}\right)$; then $\tilde{\mu}_{n}$ will be an approximate cusp sequence and Lemma 7 will apply. For ease of discussion, rotate and translate the picture so that the main cusp direction is straight upwards and the main cusp is at 0 . Without loss of generality, we consider only the right-hand side of the picture. Figure 12 shows this. We write $\phi(\tau)$ for the parameterisation of the boundary and $\psi_{+}(\tau)$ for the outer bounding $(2,3)$-cusp. Here $\tau \in \mathbb{R}$ and $\phi(\tau)$ is the point on the boundary such that $\nu_{+}=p / q+q^{2} \tau$ (see the definition of $\tau$ in the proof of Lemma 5. Section 3.1). Choosing the constants appropriately, we say that $\psi_{+}(\tau)=-a i \tau^{-2}+b \tau^{-3}$ (see $\psi_{+}$marked on Figure 15). It is easy to see from the fact that we have an $O\left(\tau^{-3}\right)$ estimate of $\phi(\tau)$ that the horizontal distance from $\phi(\tau)$ to the outer bounding curve $\psi_{+}$is $O\left(\tau^{-3}\right)$. Say this distance is less than $W \tau^{-3}$.

Before proceeding with the construction of the arc, we slightly improve Lemma 7. The proof of this lemma gives the idea of the construction of the arc. 


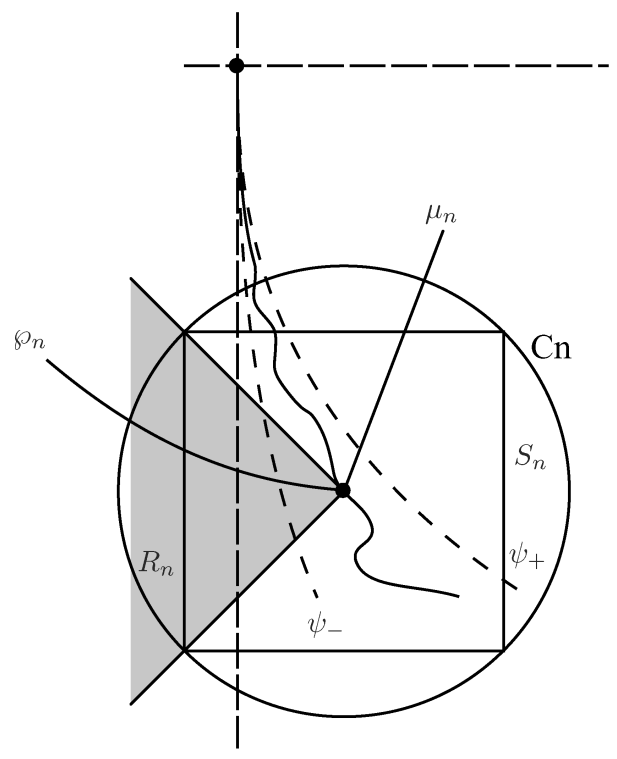

FiguRE 16. Impossible consequence of $\delta=-1$ in Equation (3.14)

Lemma 9. Let $\tilde{\mu}_{n}$ be an approximate cusp sequence, and let $\operatorname{Tr}_{n}\left(\mu_{n}\right)=2 \epsilon_{n}$ (so $\left.\epsilon_{n}= \pm 1\right)$. Then

$$
\operatorname{Tr}_{n}^{\prime}\left(\tilde{\mu}_{n}\right)=\frac{\epsilon_{n} n^{3} i \operatorname{Tr}_{\infty}^{\prime}\left(\mu_{\infty}\right)}{\pi^{2}}+O\left(n^{2}\right)
$$

Notice that if we had assumed that $r / s<p / q$, then we would need to prove $\operatorname{Tr}_{n}^{\prime}\left(\tilde{\mu}_{n}\right)=\frac{-\epsilon_{n} n^{3} i \operatorname{Tr}_{\infty}^{\prime}\left(\mu_{\infty}\right)}{\pi^{2}}+O\left(n^{2}\right)$. The point is that if the cusp neighbours are on the right-hand side of the main cusp, then they point out one way, and if they are on the left, they point out the other way (either way, they point away from the axis of the main cusp).

Proof. Without loss of generality, suppose $\epsilon_{n}=1$. In this case the initial direction of the pleating ray is $1 / \operatorname{Tr}_{n}^{\prime}\left(\mu_{n}\right)$. If $\epsilon_{n}=-1$, then it would be $-1 / \operatorname{Tr}_{n}^{\prime}\left(\mu_{n}\right)$. Let

$$
\operatorname{Tr}_{n}^{\prime}\left(\tilde{\mu}_{n}\right)=\frac{\delta n^{3} i \operatorname{Tr}_{\infty}^{\prime}\left(\mu_{\infty}\right)}{\pi^{2}}+O\left(n^{2}\right)
$$

for $\delta= \pm 1$. Suppose we have that $\delta=-1$. This leads to the contradiction, illustrated in Figure 16, that the pleating ray leaves the slice.

We can certainly say that $|\operatorname{Im} \phi(\tau)| \leq W \tau^{-3}$ for some constant $W$. Define $C_{\tau}$ to be the disc of radius $\sqrt{2} W \tau^{-3}$ about $\phi(\tau)$ so that the inscribed square $S_{\tau}$ intersects the imaginary axis. Now, for any point $\tilde{\mu}_{n} \in C_{n}$ we have that $\tilde{\mu}_{n}=$ $\mu_{n}+O\left(n^{-3}\right)$ because the radius of $C_{n}$ is $O\left(n^{-3}\right)$, and so any sequence of $\tilde{\mu}_{n} \in C_{n}$ is an approximate cusp sequence. Here $C_{n}$ is defined to be $C_{\tau}$ for the value of $\tau$ corresponding to $n$, the precise value is $\tau=n+s / q$ (see the definition of $\tau$ in the proof of Lemma 5). Lemma 7 then says that Equation (3.14) will hold for any $\tilde{\mu}_{n} \in C_{n}$.

Choose $N$ large enough so that for $n \geq N$ we have that the angle from the horizontal of $\wp_{n}^{\prime}=1 / \operatorname{Tr}_{n}^{\prime}\left(\tilde{\mu}_{n}\right)$ is less than $\pi / 4$. Let $R_{n}$ be the conical region of angle $\pi / 4$ with the horizontal, centered at the $A^{n} B$ cusp $\mu_{n}$, expanding in the direction 


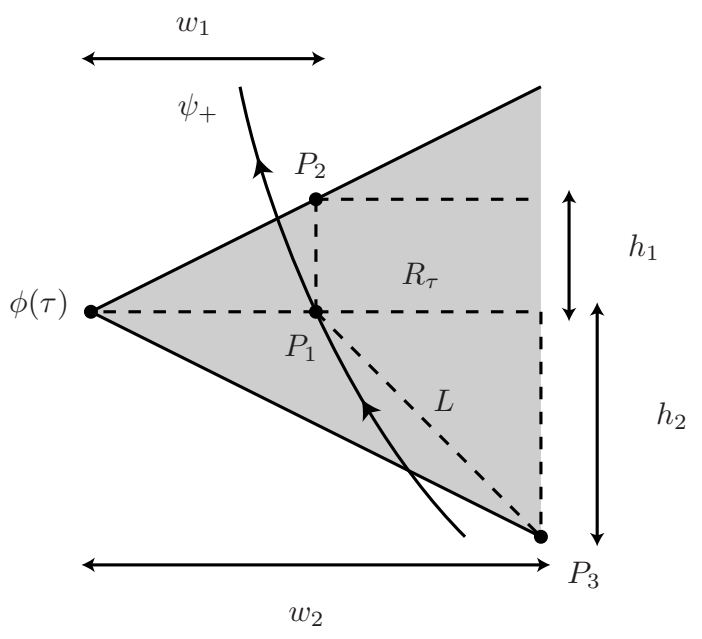

Figure 17. Convexity argument

of the negative horizontal axis. Now, $\wp_{n}^{\prime}(t)=1 / \operatorname{Tr}_{n}^{\prime}\left(\wp_{n}(t)\right)$. Since $1 / \operatorname{Tr}_{n}^{\prime}\left(\wp_{n}(s)\right)$ has angle less than $\pi / 4$ to the horizontal as long as $\wp_{n}(s) \in R_{n}$, the intersection of $\wp_{n}$ with $C_{n}$ is contained within $R_{n}$ (if $\wp_{n}$ left the region $R_{n}$, it would have a tangent with angle greater than $\pi / 4$ with the horizontal at that point). In fact, it is possible that $\wp_{n}$ could leave the region $R_{n}$ and subsequently come back, but we need only consider the initial segment of $\wp_{n}$. More precisely then, the connected component of the intersection of $\wp_{n}$ with $C_{n}$ containing the cusp $\mu_{n}$ is contained within $R_{n}$. In particular, since $S_{n}$ intersects the negative imaginary axis, so must $\wp_{n}$ (otherwise it would remain within a bounded region of the cusp). However, points on the negative imaginary axis are never inside the slice, whereas points on $\wp_{n}$ are always within the slice. This contradiction tells us that $\delta=1$ in Equation (3.14).

The construction of the curve from the $A^{n} B$ cusp to the main cusp works in much the same way. However, rather than joining the $A^{n} B$ cusp to the imaginary axis via the initial segment of the pleating ray, we join it to the outer bounding $(2,3)$ cusp via the pleating ray, and from there to the main cusp via the outer bounding $(2,3)$-cusp. Consider Figure 17, The conical region $R_{\tau}$ shown shaded is defined to be centred at $\phi(\tau)$ and bounded by two lines with gradient $\pm m_{1}$ where $m_{1}$ is any positive number. $R_{\tau}$ is expanding in the direction of the positive horizontal axis. We want to show that:

Lemma 10. For $\tau$ large enough, and for some constant $D$, the conical region $R_{\tau}$ always intersects the outer bounding curve $\psi_{+}$within a distance $D \tau^{-3}$ of $\phi(\tau)$.

Proof. We know that the outer bounding curve is $\psi_{+}(\tau)=-a i \tau^{-2}+b \tau^{-3}$ (for some $a, b \in \mathbb{R})$. It is important to note here that the parameterisations of $\psi_{+}(\tau)$ and $\phi(\tau)$ are both such that as $\tau$ increases to $\infty$, the point gets closer and closer to $\mu_{\infty}$. In Figure 17 this is illustrated by arrows on the $\psi_{+}$curve. In particular, keep this in mind when thinking about the gradients of tangents to this curve. Differentiating $\psi_{+}(\tau)$, we get that $\psi_{+}(\tau)^{\prime}=2 a i \tau^{-3}-3 b \tau^{-4}$. This gives the gradient from the 
horizontal of the tangent line a value $m(\tau)=-2 a \tau / 3 b$. Since this tends to $-\infty$ as $\tau$ increases, we can say that for $\tau>T_{1}$, we have $|m(\tau)|>m_{2}$ for a constant $m_{2}$ which we will choose to be anything larger than $m_{1}$ (we could choose $m_{i}=i$ for instance).

On Figure 17 the point labelled $P_{1}$ is the intersection of $\psi_{+}$with the horizontal line through $\phi(\tau)$. The point labelled $P_{2}$ is the point on the intersection of the vertical line through $P_{1}$ and the upper bounding line of the cone. The point labelled $P_{3}$ is the intersection of the line $L$, which is from $P_{1}$ with gradient $-m_{2}$, with the lower bounding line of the cone. We define $w_{1}$ to be the distance from $\phi(\tau)$ to $P_{1}$, $h_{1}$ to be the distance from $P_{1}$ to $P_{2}, w_{2}$ to be the distance from $\phi(\tau)$ to the vertical line through $P_{3}$, and $h_{2}$ to be the distance from $P_{3}$ to the horizontal line through $\phi(\tau)$.

We get that $h_{1} / w_{1}=h_{2} / w_{2}=m_{1}$ and $h_{2} /\left(w_{2}-w_{1}\right)=m_{2}$. This gives $h_{1}=$ $m_{1} w \leq m_{1} W \tau^{-3}$ and $h_{2}=w /\left(1 / m_{1}-1 / m_{2}\right) \leq W \tau^{-3} /\left(1 / m_{1}-1 / m_{2}\right)$. We want to define a constant $T_{2}$ such that if $\tau>T_{2}$ and for some other value, say $\tilde{\tau}$, we have that $\operatorname{Im} \psi_{+}(\tilde{\tau})<\operatorname{Im} \phi(\tau)-h_{2}$ (that is, $\psi_{+}(\tilde{\tau})$ is below a horizontal line through the point $P_{1}$ in Figure [17), then we have that $\tilde{\tau}>T_{1}$. This can easily be arranged using the formula for $\psi_{+}$and the estimate for $\operatorname{Im} \phi(\tau)$. Now for any $\tau>T_{2}$, because $|m(\tilde{\tau})|>m_{2}$ whenever $\operatorname{Im} \psi_{+}(\tilde{\tau})>\operatorname{Im} \phi(\tau)-h_{2}$, the upper (resp. lower) bounding line of the cone intersects $\psi_{+}$within a distance $\sqrt{w_{1}^{2}+h_{1}^{2}}$ (resp. $\sqrt{w_{2}^{2}+h_{2}^{2}}$ ), the distance from $\phi(\tau)$ to $P_{2}$ (resp. $P_{3}$ ). This gives us the required constant $D$.

Now we proceed to construct a curve as in the proof of Lemma 9 For all sequences of points $\tilde{\mu}_{n} \in C_{n}$ (the circle centred around the $n$th neighbouring cusp) we have that $\tilde{\mu}_{n}$ is an approximate cusp sequence. Choose $N$ large enough that the absolute value of the gradient of a line in the direction $1 / \operatorname{Tr}_{n}^{\prime}$ is smaller than $m_{1}$ for $n \geq N$. Points on $\wp_{n}$ within a distance $D / n^{3}$ of the $n$th cusp must be within the conical region $R_{n}$ which intersects the outer bounding $(2,3)$-cusp $\psi_{+}$, so the pleating ray must also intersect $\psi_{+}$. (As before, we are only interested in the connected component of $\wp_{n}$ intersected with $C_{n}$ containing $\mu_{n}$, this initial segment of $\wp_{n}$ must be contained in $R_{n}$.) This gives us the curve we wanted, just connect the initial segment of $\wp_{n}$ from the $n$th cusp to its intersection with $\psi_{+}$to the segment of $\psi_{+}$from this intersection point to the main cusp.

\subsection{Main theorems.}

Proof of Theorem 1. Given a point on the boundary, connect the base point $z_{0}$ to any very close cusp $z_{1}$ by a curve $\alpha_{0}$ as in the definition of spiralling in Section 2.2. Let $z_{2}$ be a very close neighbouring cusp of $z_{1}$ on the right-hand side. Construct the curve $\alpha_{1}$ by joining to the end of $\alpha_{0}$ the two segments described above (the initial segment of the pleating ray coming out of $z_{2}$ followed by the outer bounding $(2,3)$ cusp of the $z_{1}$ cusp). Let $L_{1}$ and $L_{2}$ be the branches of the $\log$ function $L$, in the definitions in Section 2.2 corresponding to $z_{1}$ and $z_{2}$. Now $L_{1}(z)-L_{2}(z)=\log \frac{z-z_{1}}{z-z_{2}}$. So, if we look at only those points on $\alpha_{1}$ and $\alpha_{2}$ which don't get too close to $z_{1}$ or $z_{2}$ (say, exclude a small $\eta$-neighbourhood of $z_{1}$ so that $\left|z_{2}-z_{1}\right|<\eta / \kappa$ for some large $\kappa$ ), we can make $L_{1}\left(\alpha_{1}(t)\right)-L_{2}\left(\alpha_{2}(t)\right)$ as small as we like by making $\eta$ small and $\kappa$ large. This is because $\left|\frac{z-z_{1}}{z-z_{2}}-1\right|=\left|\frac{z_{2}-z_{1}}{z-z_{2}}\right| \leq 1 / \kappa, \log (1)=0$ and $\log$ is continuous at 1 . Note that since we are choosing an arbitrarily close neighbour $z_{2}$ of $z_{1}$ we can ensure that $z_{2}$ is in the $\eta$-neighbourhood of $z_{1}$ that we choose. It will be important in the proof of Theorem 2 that there are a countably infinite number 
of choices for the point $z_{2}$, any of the countably infinitely many cusp neighbours within the $\eta$-neighbourhood of $z_{1}$ will do.

Suppose then that except in this neighbourhood of $z_{1}$ we have that $\mid L_{1}\left(\alpha_{1}(t)\right)$ $L_{2}\left(\alpha_{2}(t)\right) \mid<\delta$ for some small $\delta$, say $\delta=\pi / 24$. Now, we know what $\alpha_{1}$ and $\alpha_{2}$ look like in this small neighbourhood of $z_{1}$, and we can easily see that $\operatorname{sp}$. $\operatorname{deg} \alpha_{2} \leq$ sp. $\operatorname{deg} \alpha_{1}-\pi / 3+2 \delta$. This gives us that sp. $\operatorname{deg} \alpha_{2} \leq$ sp. $\operatorname{deg} \alpha_{1}-\pi / 4$. Repeating this procedure, we get that sp. $\operatorname{deg} \alpha_{n} \leq \mathrm{sp} . \operatorname{deg} \alpha_{1}-n \pi / 4$ and, in particular, that sp. $\operatorname{deg} \alpha_{n} \rightarrow-\infty$ as $n \rightarrow \infty$.

So the boundary spirals to an indefinite extent near every point. By choosing neighbouring cusps on the left-hand side instead of the right, we can find curves spiralling arbitrarily clockwise instead of counterclockwise, and by suitably alternating our choices we can keep the degree of spiralling bounded.

Proof of Theorem 2. Let $z_{\infty}=\lim z_{n}$ where the $z_{n}$ are the series of cusp points in the previous proof (in fact, we will add an additional requirement on $z_{n}$ later in this proof). Writing $L_{n}(z)$ as before, and $L_{\infty}(z)=\log \left(z-z_{\infty}\right)$ defined in the same way, we get $L_{\infty}(z)-L_{n}(z)=\log \left(1+w_{n}(z)\right)$ where $w_{n}(z)=\left(z_{n}-z_{\infty}\right) /\left(z-z_{n}\right)$. If we can find a sequence $\zeta_{n}$ such that $\zeta_{n} \rightarrow z_{\infty}, \operatorname{Im} L_{n}\left(\zeta_{n}\right)$ is unbounded and $\log \left(1+w_{n}\left(\zeta_{n}\right)\right)$ is bounded, then $\operatorname{Im} L_{\infty}\left(\zeta_{n}\right)$ will be unbounded, which would prove that $z_{\infty}$ was a point of infinite spiralling on the boundary. To show that $\log \left(1+w_{n}\left(\zeta_{n}\right)\right)$ is bounded it is enough to find a sequence such that $\left|w_{n}\left(\zeta_{n}\right)\right|<1 / 2$, or equivalently that $\left|\zeta_{n}-z_{n}\right|>2\left|z_{n}-z_{\infty}\right|$. For $\left|z-z_{n}\right| \leq \eta$, for some $\eta>0$ depending on $n$, we know that $\operatorname{Im} L_{n}(z)>a+b n$ for some constants $a, b>0$. (This just follows from the previous proof.) Now, if $\left|z_{n}-z_{\infty}\right|<\eta / 2$, then we can choose a point $\zeta_{n}$ a distance $\eta$ from $z_{n}$, and this sequence satisfies $\left|w_{n}\left(\zeta_{n}\right)\right|<1 / 2$. The additional requirement on $z_{n}$ mentioned at the start of this proof is that $\left|z_{n}-z_{\infty}\right|<\eta / 2$ which can be ensured by always choosing the next neighbouring cusp in the sequence $\left(z_{n}\right)$ sufficiently close to the previous one.

The set of points where the spiralling is infinite is clearly dense (this procedure can be started as close to any point as you like). Moreover, the number of limit points you can get to is uncountable. For any given choice of the first $m$ points in the sequence $\left(z_{n}\right)$, there is a countably infinite number of choices for the next point $z_{m+1}$ (this is the comment in the proof of Theorem 1 that says there are a countably infinite number of choices for what was called $z_{2}$ in that proof). Here we will assume that the point $z_{1}$ is common to all such sequences. Even with this restriction we get an uncountable number of limit points. Each choice of sequence $\left(z_{n}\right)$ gives rise to a unique limit $z_{\infty}$. This is because the continued fraction expansion of an irrational is unique. Given a sequence $\left(z_{n}\right)$, define the sequence $\left(r_{n}\right)$ to be the rational numbers associated to the cusps (so that $z_{n}=\mu\left(r_{n}\right)$ ). The sequence $\left(r_{n}\right)$ will be the sequence of continued fraction partial approximates to $\beta=\lim r_{n}=\mu^{-1}\left(z_{\infty}\right)$ (because $z_{n+1}$ is a neighbour of $z_{n}$ ). Since each irrational $\beta$ has a unique continued fraction approximation, different sequences will give rise to different limits. Let $S$ be the set of limits $z_{\infty}$ coming about in this way, we have shown that there is a bijection between $S$ and the set of countably infinite sequences of natural numbers $\mathbb{N}^{\mathbb{N}}$. Explicitly, this bijection is as follows. Given a finite sequence $\left(z_{n}\right)_{n=1}^{m}$, there is a countably infinite set $Z\left(\left(z_{n}\right)_{n=1}^{m}\right)$ of choices for the next point $z_{n+1}$. For each such sequence, choose a bijection $\pi\left(\left(z_{n}\right)_{n=1}^{m}\right): Z\left(\left(z_{n}\right)_{n=1}^{m}\right) \rightarrow \mathbb{N}$. Given a sequence $\left(a_{n}\right) \in$ $\mathbb{N}^{\mathbb{N}}$, we define a sequence $z_{n}$ inductively as follows. The first point $z_{1}$ is always the same. Given the first $m$ points, $\left(z_{n}\right)_{n=1}^{m}$ we define $z_{n+1}=\pi\left(\left(z_{n}\right)_{n=1}^{m}\right)^{-1}\left(a_{n}\right)$. The 
bijection between $\mathbb{N}^{\mathbb{N}}$ and $S$ sends a sequence $\left(a_{n}\right) \in \mathbb{N}^{\mathbb{N}}$ to the limit $z_{\infty}$ of this sequence. This is surjective by definitive, and injective because such sequences give rise to unique limits. Since $\mathbb{N}^{\mathbb{N}}$ is uncountable, so is $S$. The character of the set of points about which this result proves there is infinite spiralling, is somewhat akin to a countable union of Cantor sets.

\section{REFERENCES}

[CCHS03] Richard Canary, Marc Culler, Sa'ar Hersonsky and Peter Shalen, Approximation by maximal cusps in boundaries of deformation spaces of Kleinian groups, J. Differential Geom. 64 (2003), no. 1, 57-109. MR2015044 (2004j:57020)

[ChoiSeries06] Young Choi and Caroline Series, Lengths are coordinates for convex structures, J. Differential Geom. 73 (2006), pp. 75-117. MR2217520

[Culler86] Marc Culler, Lifting representations to covering groups, Adv. in Math. 59 (1986), no. 1, 64-70. MR0825087 (87g:22009)

[KeenSeries93] Linda Keen and Caroline Series, Pleating coordinates for the Maskit embedding of the Teichmüller space of punctured tori, Topology 32 (1993), no. 4, 719-749. MR1241870 (95g:32030)

[KeenSeries04] Linda Keen and Caroline Series, Pleating invariants for punctured torus groups, Topology 43 (2004), no. 2, 447-491. MR2052972 (2005f:30077)

[McMullen91] Curt McMullen, Cusps are dense, Ann. of Math. (2) 133 (1991), no. 1, 217-247. MR:1087348(91m:30058)

[McMullen98] Curt McMullen, Complex earthquakes and Teichmüller theory, J. Amer. Math. Soc. 11 (1998), no. 2, 283-320. MR.1478844(98i:32030)

[Minsky99] Yair Minsky, The classification of punctured-torus groups, Ann. of Math. (2) 149 (1999), no. 2, 559-626. MR 1689341 (2000f:30028)

[Pomm92] Christian Pommerenke, Boundary behaviour of conformal maps, Grundlehren der Mathematischen Wissenschaften [Fundamental Principles of Mathematical Sciences], 299. Springer-Verlag, Berlin, 1992. MR1217706 (95b:30008)

[Miyachi03] Hideki Miyachi, Cusps in complex boundaries of one-dimensional Teichmüller space, Conform. Geom. Dyn. 7 (2003), 103-151. MR2023050 (2004j:30091)

[Wright88] David Wright, The shape of the boundary of Maskit's embedding of the Teichmüller space of once-punctured tori, preprint.

68 New Street, Leamington Spa, CV31 1HL, United Kingdom

E-mail address: goodman@maths.warwick.ac.uk

Current address: 73 Huddleston Road, London, N7 0AE, United Kingdom

E-mail address: dan.goodman@cantab.net 